\title{
One-dimensional, Two-phase Flow Modeling Toward Interpreting Motor Slag Expulsion Phenomena
}

\author{
Tim Kibbey, Jacobs Technology \\ Jacobs ESTS Group
}

Aluminum oxide slag accumulation and expulsion was previously, ${ }^{\mathrm{i}, \mathrm{ii}, \mathrm{iii}}$ shown to be a player in various solid rocket motor phenomena, including the Space Shuttle's Reusable Solid Rocket Motor (RSRM) pressure perturbation, or "blip," and phantom moment. In the latter case, such un-commanded side accelerations near the end of burn have also been identified in several other motor systems. However, efforts to estimate the mass expelled during a given event have come up short. Either bulk calculations are performed without enough physics present, or multiphase, multidimensional Computational Fluid Dynamic analyses are performed that give a snapshot in time and space but do not always aid in grasping the general principle.

One-dimensional, two-phase compressible flow calculations yield an analytical result for nozzle flow under certain assumptions. This can be carried further to relate the bulk motor parameters of pressure, thrust, and mass flow rate under the different exhaust conditions driven by the addition of condensed phase mass flow. An unknown parameter is correlated to airflow testing with water injection ${ }^{\text {iv }}$ where mass flow rates and pressure are known. Comparison is also made to full-scale static test motor data where thrust and pressure changes are known and similar behavior is shown.

The end goal is to be able to include the accumulation and flow of slag in internal ballistics predictions. This will allow better prediction of the tailoff when much slag is ejected and of mass retained versus time, believed to be a contributor to the widely-observed "flight knockdown" parameter.

\section{Two-Phase Flow Model}

Previous analyses ${ }^{i v, v}$ have treated the slag flow as a monolithic inert mass partially blocking the nozzle as it flows overboard. These analyses estimated the momentum of the inert mass but do not account for the momentum reduction of the gas required to accelerate the inert mass, thus violating conservation of momentum. Since the pooled slag is thought to be a liquid-gas slurry, and to strongly interact with the upstream gas flow, perhaps a more diffuse model of interaction is appropriate. This is especially true if the slag does attain significant momentum when flowing through the nozzle, which would be debited from the gas flow.

A one-dimensional two-phase compressible flow model was derived for solid rocket motor nozzle flows by Kliegel $\mathrm{l}^{\mathrm{vi}}$. The analysis assumes full momentum and convective thermal coupling between phases, but no particle-to-particle interaction; thus particles can be either liquid or solid. It also assumes an ideal, inviscid gas, except for drag on the particles, of constant properties and no chemical reactions. An analytic derivation is possible assuming spherical particles in Stokes flow and a constant fractional lag, meaning as the flow accelerates through the nozzle, the particles' velocity is always the same fraction of the gas velocity at the same location. A discussion of how valid an approximation this might be of actual non-uniform behavior is reserved for a later section.

\section{Derivation}


Kliegel's solution is presented in Equations Error! Reference source not found. through Error! Reference source not found., showing the same form as isentropic flow, but with modified Mach number $(\bar{M})$ and specific heats ratio $(\bar{\gamma})$. This includes the following parameter definitions:

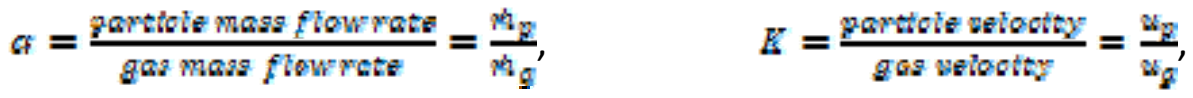

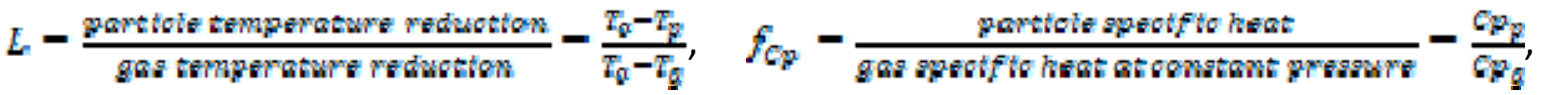

$$
\begin{aligned}
& B=\frac{\vdots+\alpha B^{2}}{1+\alpha / C q^{2}} \\
& C=1+\alpha\left[K(\gamma(1-K)+K)+(\gamma-1) f_{S y} E\right] \\
& \text { In } \sqrt{\bar{C}} M \\
& 7-1=(y-1) \frac{2}{9} \\
& \frac{1-\varepsilon}{E}=3 F r f_{C y} \frac{1-E}{R} \\
& h(\bar{M})=1+\frac{\bar{r}-1}{2} \bar{M}^{2}, \quad \frac{\tau_{0}}{T_{y}}=h(\bar{M}), \quad \frac{E_{g}}{p}=(h(\bar{M})) \frac{F}{\bar{F}-1}
\end{aligned}
$$

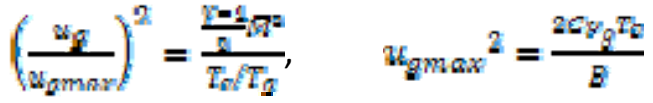

$$
\begin{aligned}
& \frac{\theta_{n}}{\beta_{2}}=(h(h))+
\end{aligned}
$$

Note that, when $\mathrm{K}=\mathrm{L}=1$, this reduces to the heavy gas assumption ${ }^{\mathrm{vii}}$. One result is that the transformed mixture Mach number, $\bar{M}$, is 1 at the geometric throat, while the gas Mach number, $\mathrm{M}$, is less than 1 . Note that this applies also to standard RSRM flow, where the heavy gas reduction is typically used for ballistic analysis. Herein, when referring to the "RSRM gas" these heavy gas properties will be used. A future analysis could attempt to derive the equations with multiple particle sizes subject to multiple lag ratios representing smoke, larger particles in the flow, and pooled slag ejecta to see if higher fidelity can be achieved.

Now, a change in characteristic velocity $\left(c^{*}\right)$ due to the addition of condensed flow can be derived, which will tie mass flow rate to chamber pressure. Then, a change in thrust coefficient $\left(C_{f}\right)$ can be derived, tying in thrust and Specific Impulse (Isp).

\section{Connecting mass flow to pressure, $\mathrm{c}^{*}$}

Consider mass flow rate at the nozzle throat, invoking the ideal equation of state for the gas phase and Equation (3) with continuity, resulting in Equation Error! Reference source not found..

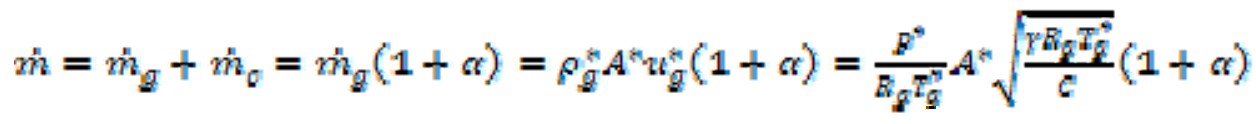


Substituting Equation (6) puts Equation Error! Reference source not found. in terms of chamber conditions.

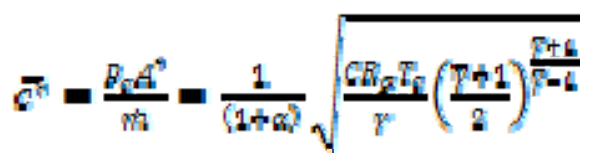

Note the appearance of both gas $\gamma$ and mixture $\bar{\gamma}$ in Equation (9), and its similarity to the standard gas $\mathrm{c}^{*}$ with the addition of $C$ and $(1+\alpha)$.

The goal is to show a chamber pressure increase due to addition of condensed flow. Given condition 1 with only gas flow and condition 2 with the same gas flow rate plus condensed flow, the ratio of pressure 2 to pressure 1 is given in Equation (10). This is the appropriate form to apply to the air/water flow data below.

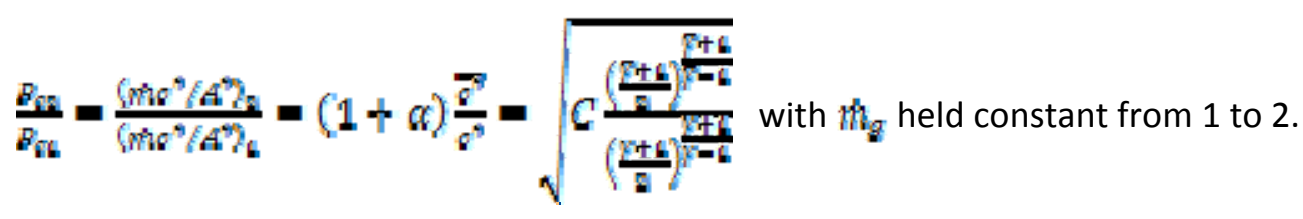

\section{Connecting thrust to pressure, $\boldsymbol{C}_{f}$}

The steady-state momentum balance gives the thrust equation Error! Reference source not found., here assuming vacuum conditions.

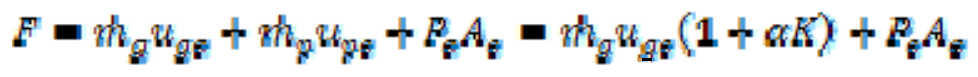

With $m_{g}=\frac{\frac{\omega}{6}}{(1+\alpha)}$, Equation Error! Reference source not found. substitutes $m_{g}$, and the Equation (7) substitutes gas velocity.

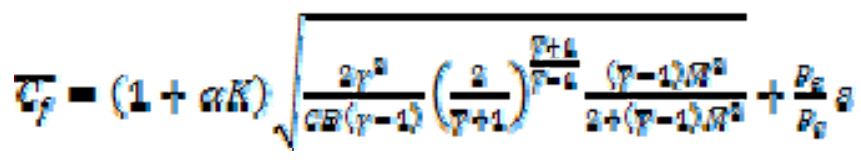

Now the thrust ratio can be solved, with Equation Error! Reference source not found. again showing the similarity in form to the standard gas $C_{f}$.

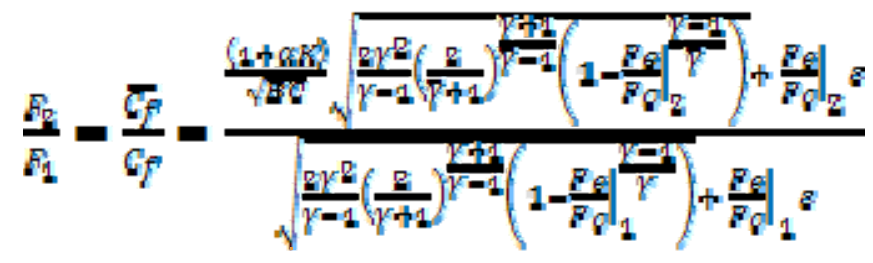




\section{General Results}

Two sets of gas and condensed phase properties were considered for demonstrating this model: air and water, consistent with Whitesides' subscale testing ${ }^{\text {iv }}$, and aluminum oxide slag with RSRM propellant combustion gas (Figure 1). In the latter case, the main combustion products are assumed to act as a heavy gas.
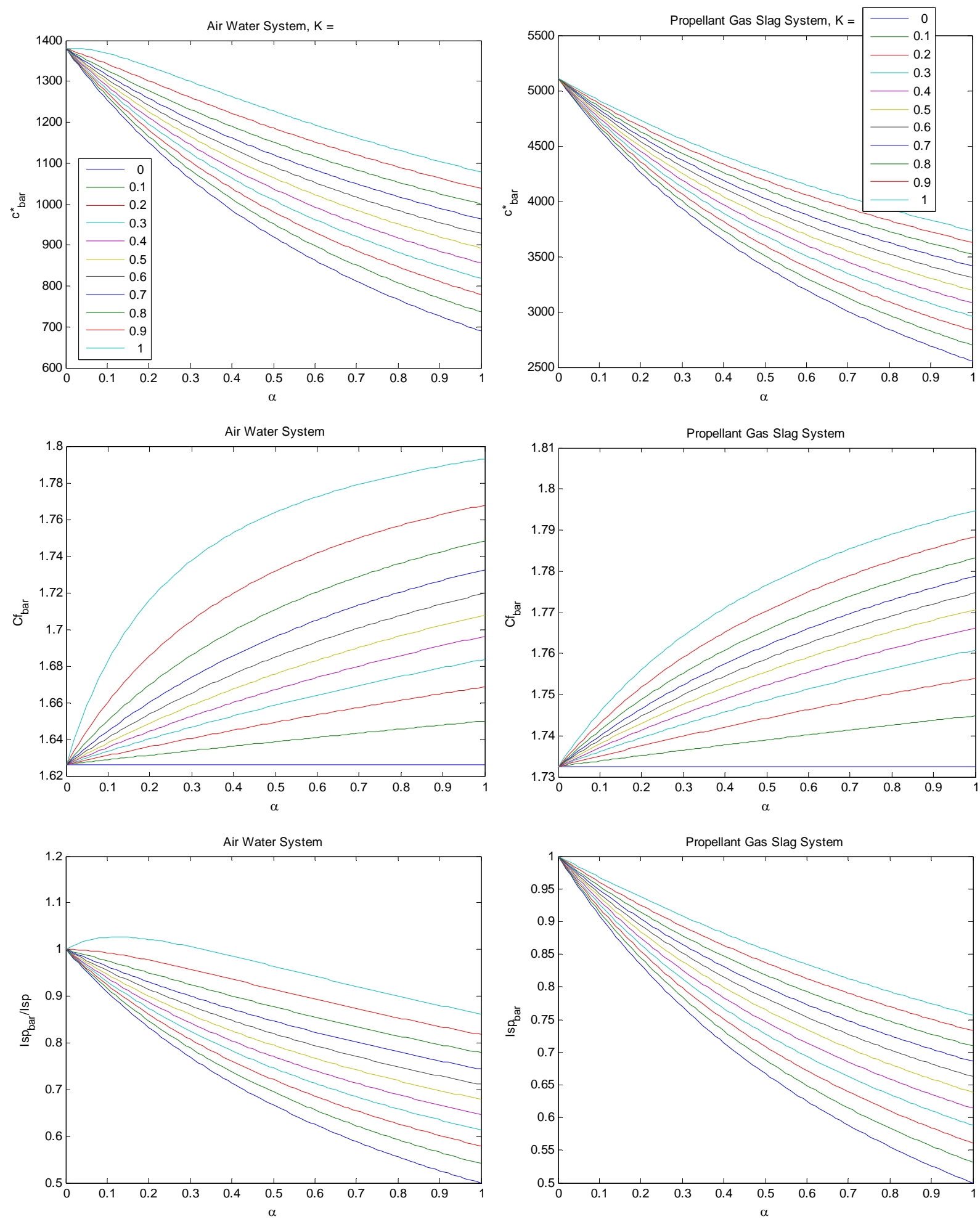

Figure 1: Two-Phase Flow Model Results for (a) Air Water, and (b) Gas Slag Properties 


\section{Calibration to Air/Water Flow data}

Computing the fractional velocity lag requires assumptions regarding particle size, viscosity, and drag coefficient that affect the drag of the gas on the condensed phase. A good starting point for estimating this behavior is the subscale air flow tests with water injection that Whitesides ${ }^{\text {iv }}$ directed at Marshall Space Flight Center. Two scaled RSRM contour nozzles were used with water injected in different ways. The most applicable test was with water injected behind the nozzle cavity, representative of where slag would collect in a solid rocket motor; this is the Main Data on Figure 2.

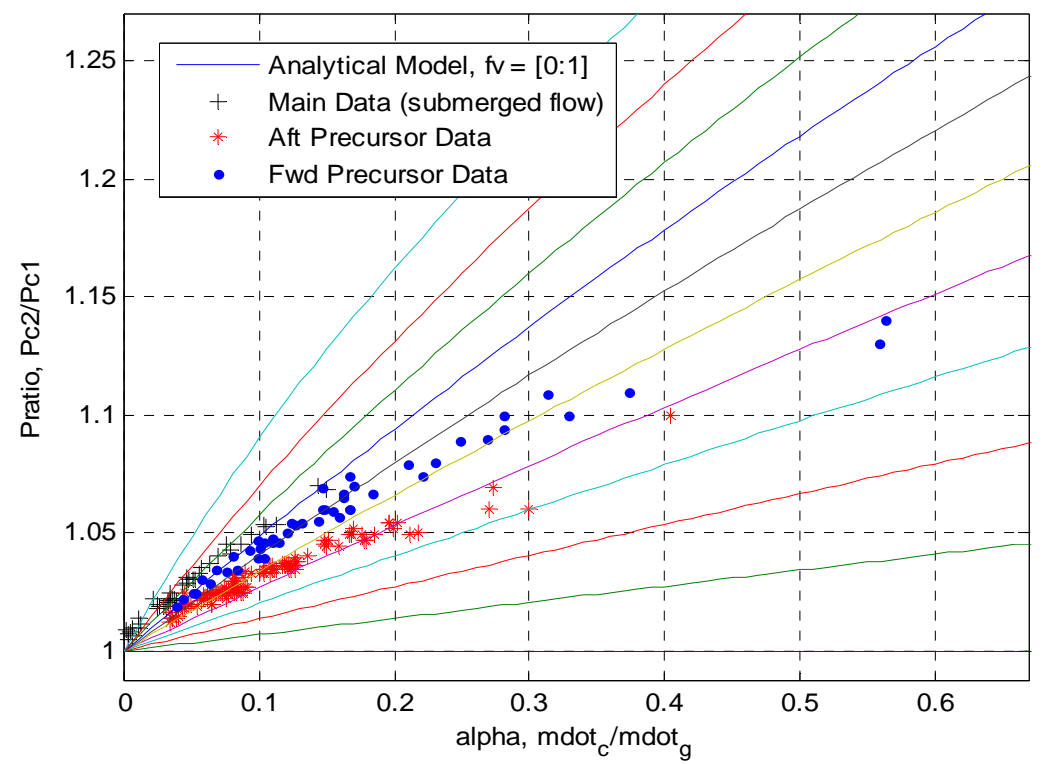

Figure 2: Water Injection Testing Correlated to Analysis

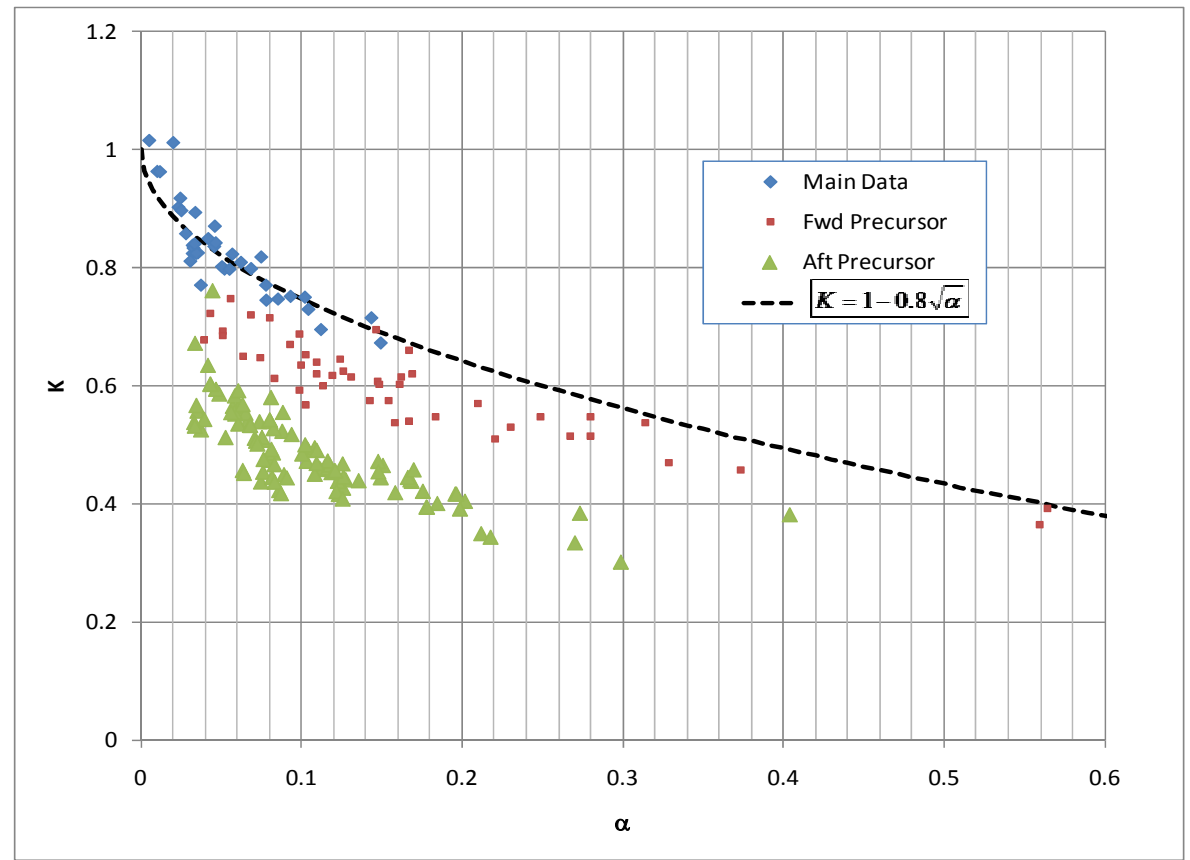

Figure 3: Determination of Velocity Lag, K, from Air-Water Data 
The data can be turned around to fit a curve relating $\mathrm{K}$ to $\alpha$. This is given in Figure 3 , where the precursor data is also shown for comparison. Clearly for full confidence, the Main Data curve fit should only be used within the data range. $\mathrm{K}$ as a function of $\alpha$ sums up all the properties related to particle drag, thus simply defining the interaction without needing to know several characteristics.

\section{Full-Scale Motor Static Test Data}

At the time of the focused RSRM pressure blip studies, it was noted by Martin iii and Cox ${ }^{\text {viii }}$ based on two static test motors that the thrust-to-pressure ratio (FoP) increased during a blip. Figure 1 shows this is consistent with the model presented herein, where the FoP increases due to the thrust coefficient increase. The database of thrust behavior during pressure blips has significantly increased since then. For the current effort, a consistent data processing and analysis method was developed to examine 25 blips from the 60-80 second timeframe on 6 motors.

\section{Blip Data Analysis}

The measured thrust, converted to vacuum, and pressure data are smoothed and the nominal trace is scaled to match the region near the pressure blip of interest. By examining these two traces, a blip start time is selected and the nominal is scaled again to match the pressure of the blip start. The ratio of actual to no-blip FoP, Equation Error! Reference source not found., can then be compared to $\frac{T_{P}}{G}$ in the two-phase flow model.

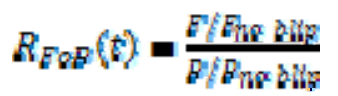

$P_{\text {no blip }}$ is just the interpolated scaled nominal versus time, but $F_{\text {no blip }}$ must be adjusted from the nominal to correct for motor variation of FoP that is unrelated to blip. This is done by computing the ratio of data FoP to nominal FoP and averaging the $1 / 2$ sec prior to the blip to establish a baseline FoP ratio, $R_{\text {FoP }}$ init. An example of this is shown in Figure 4. There is a clear quasi-steady FoP match prior to the blip start, and a clear rise in FoP during the blip, but the initial value is not 1.

To properly finish Equation Error! Reference source not found., compute the motor-specific no-blip thrust, Equation Error! Reference source not found..

$$
E_{\text {no bitp }}=\left(\text { tenterpolated } E_{\text {nomgo }}\right) * \text { FoF ractinto }
$$

One of the large blips on FSM-7 will be used as an example of the analysis. Figure 5 shows this blip with the appropriate scaling of the nominal. The above equations result in Figure 6, with the ratio of blip to no-blip FoP's on the left and the thrust rise plotted against the pressure rise on the right. In both these, the hysteresis is evidence of the thrust rise leading the pressure rise. The thrust increase rolls over approaching the quasi-steady peak of the blip, then the thrust decrease again leads on the way down. It is expected that if the fully transient behavior were modeled, the rising and falling portions of the curve would converge toward the central slope captured by the peak value. A linear fitting scheme of the rising portion was attempted to determine $R_{F o p}$ as a function of $P / P_{\text {no blip. }}$ However, this suffered the 
drawbacks of ignoring the synch-up of $(1,1)$ by using the previous half-second of data and of strong response to transient rise and spurious variation. This resulted in high data scatter when all the blips were compiled.

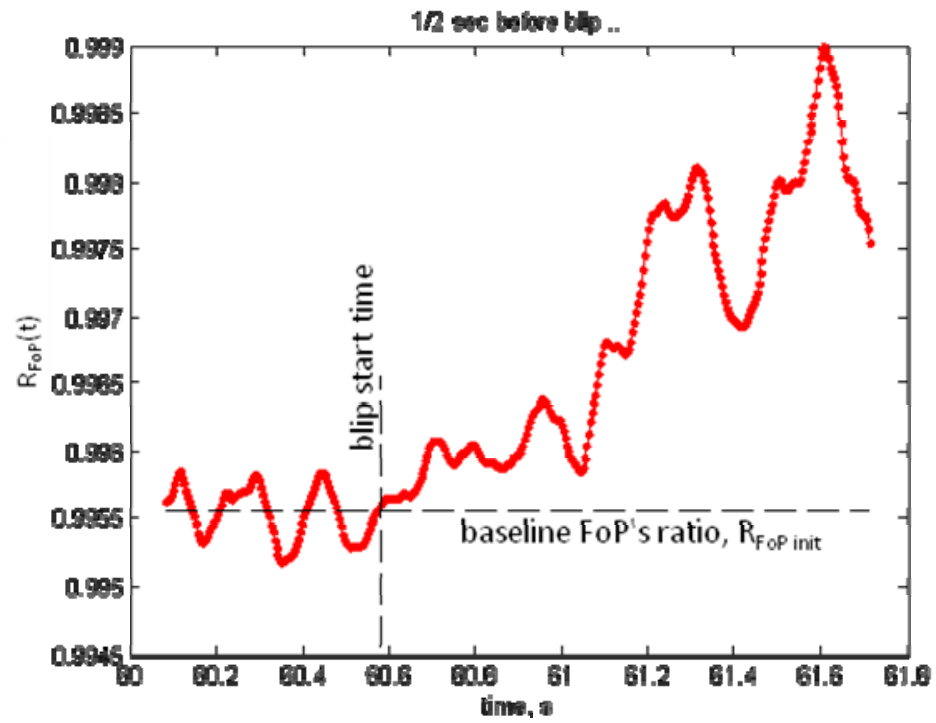

Figure 4: Flight Support Motor (FSM-) 13 FoP Baselining Example
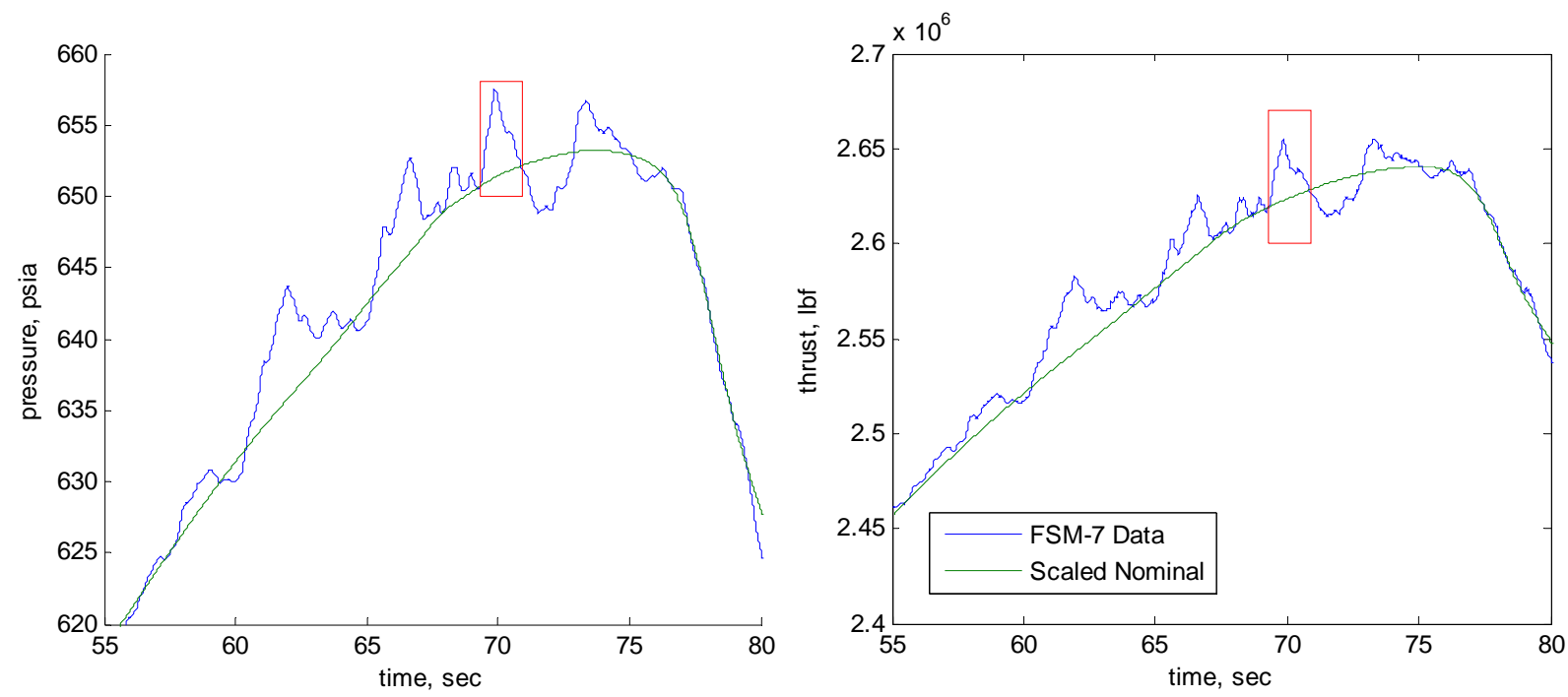

Figure 5: FSM-7 Data with Nominal Scaled for the Blip at $70 \mathrm{sec}$ 

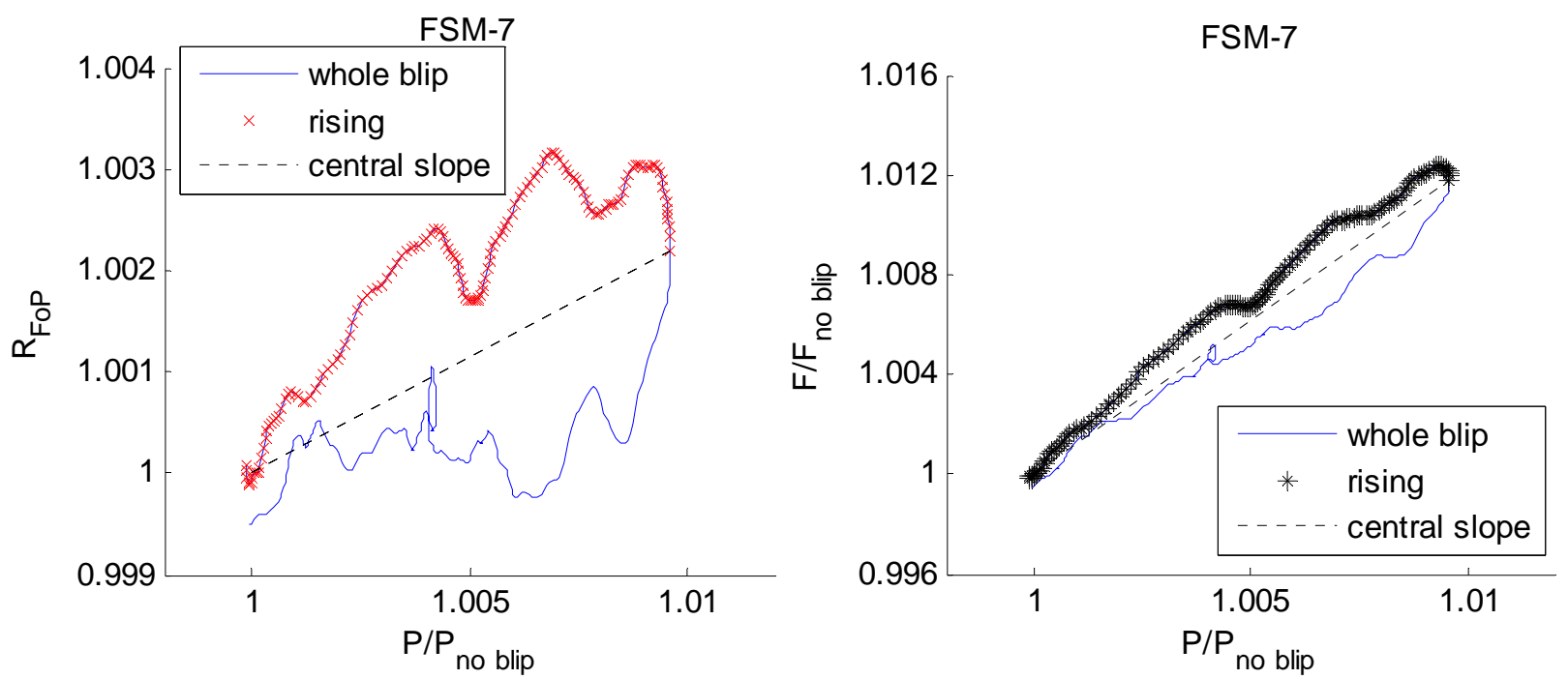

Figure 6: Processing of FSM-7's $70 \mathrm{sec}$ Blip

Having observed all the blips in this manner, the same general behavior was exhibited. And though much variation was seen in the rising portion, the peak region is quite consistent. To compile the effect, the $R_{F o p}$ at peak $P / P_{\text {no blip }}$ was selected as the $\frac{\bar{G}}{c_{i}}$ for that pressure ratio. Note that peak of blip is defined here as the maximum $\mathrm{P} / \mathrm{P}_{\text {no blip, }}$ not the local maximum pressure.

\section{Blip Thrust Coefficient Results}

The results of this analysis are presented for the 25 blips in Figure 7. The left hand figure shows a discernible correlation, perhaps linear. However, further examination of the data suggests that it could be fit better by treating as two populations shown tightly correlated in the right hand figure as high- and low-slope groups.

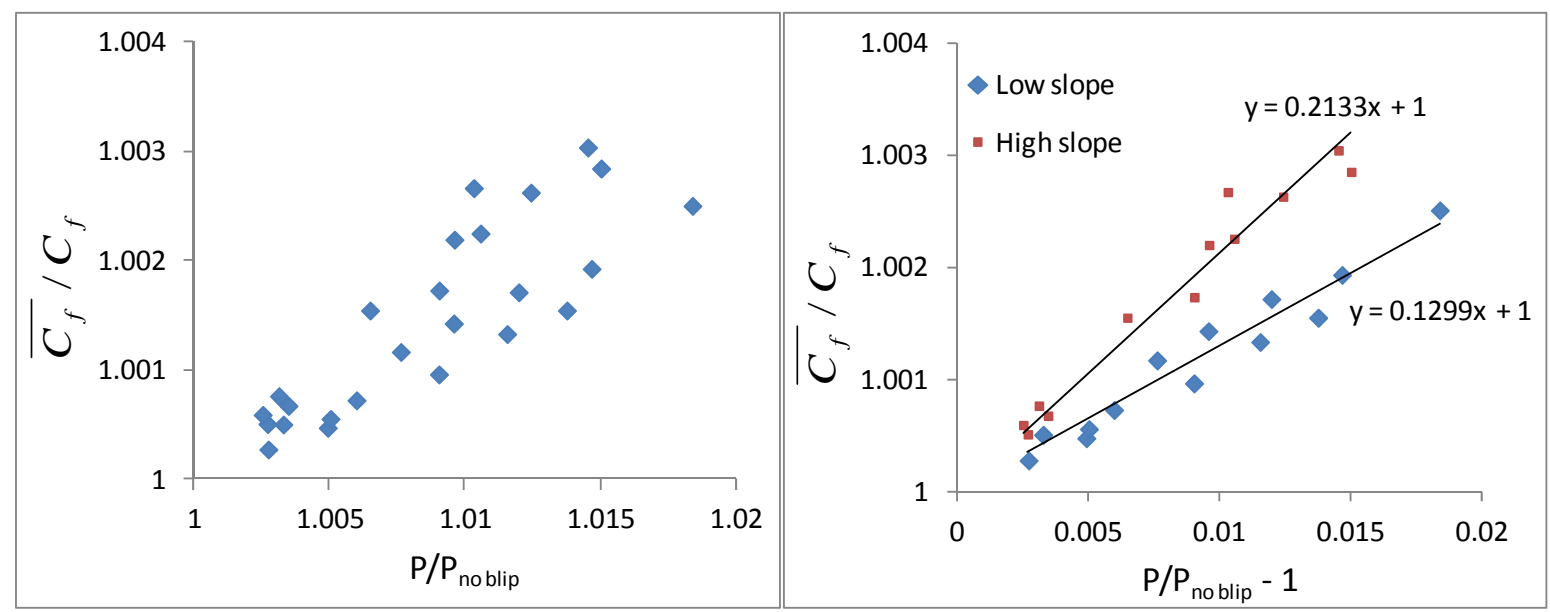

Figure 7: RSRM Blip FoP Study Results

Figure 8 examines the distribution of each point's individual $\frac{}{6}$ slope, adding further credibility to the thought of 2 populations; note the histogram's double-hump nature, and the gap in slopes around 0.16. More work would be required to identify a source for the difference between the two types. It may 
depend upon the proximity of blips to each other or the duration of the blip, perhaps similar to Hopson's" observation about "slosh" and "spill family" events on flight motors.

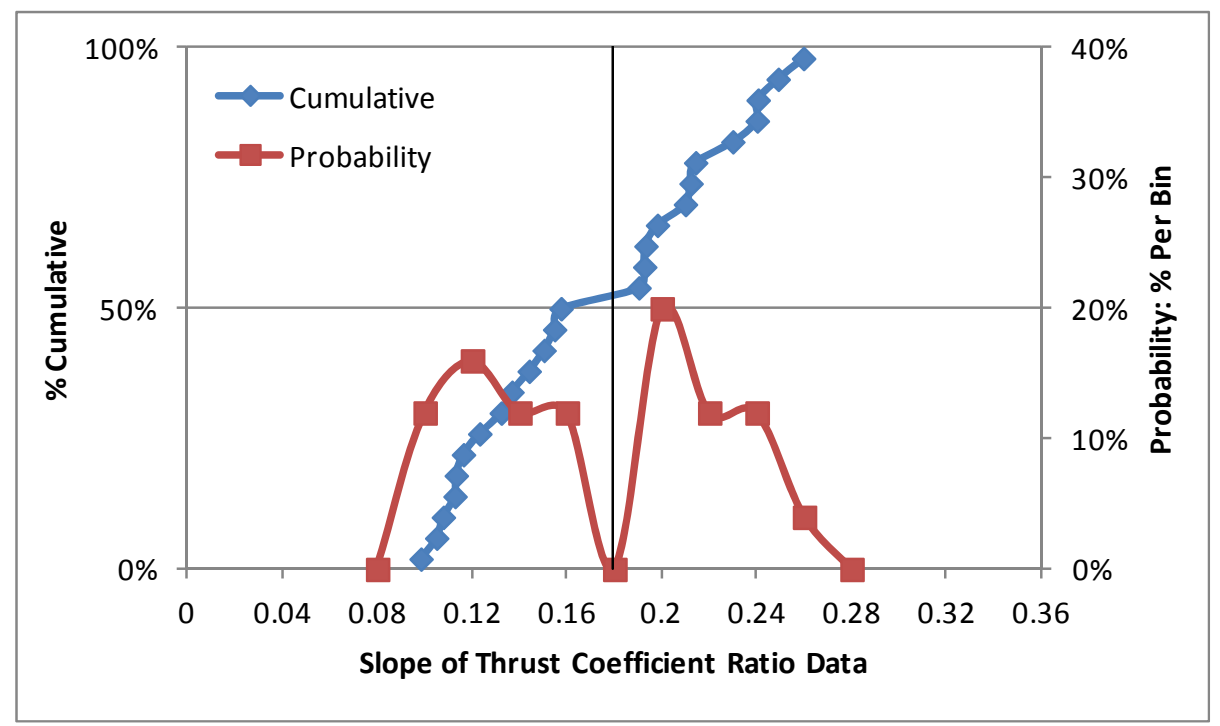

Figure 8: Histogram and Cumulative Distribution of Slopes

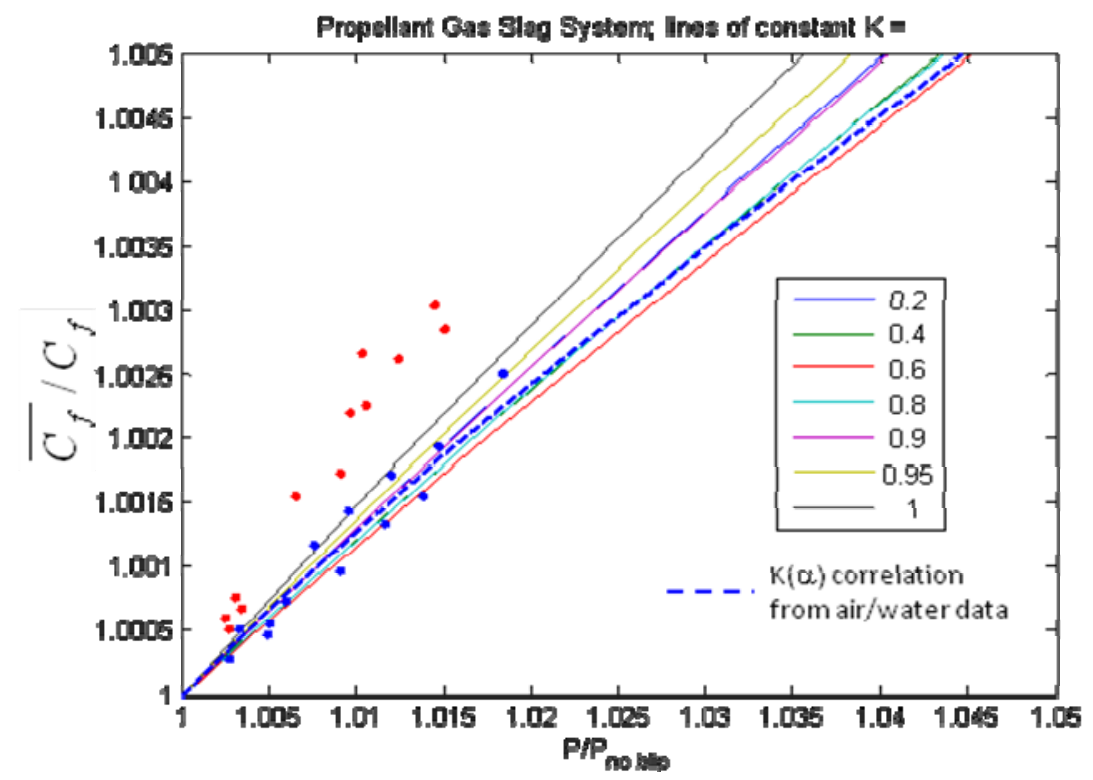

Figure 9: Motor Blip Data with Two-Phase Flow Model

Now these data can be compared to the prediction of the two-phase flow model using properties for the RSRM gas/slag system. They are plotted together in Figure 9, where the lower slope family of points coincides quite well with the model. Note that the range of the model for different $\mathrm{K}$ values is more compressed here plotted against $P / P_{\text {no blip }}$ than when plotted against $\alpha$. This is both good and bad. The narrow channel of the model solutions being lined up with the data lends credence to this model as a good bulk approximation of the phenomenon. However, given the noise level of the data, it neither corroborates nor discounts the correlation of $K$ developed from the air/water test data.

\section{Summary and Conclusions}


An analytic solution is available for 1D, 2-phase compressible flow under certain assumptions.

Water/airflow testing performed at MSFC in the 1990s calibrates the solution by setting coefficients so that a link between pressure, thrust, and mass flow rate excursions due to slag expulsion can be estimated. In addition, the set of full-scale shuttle motor thrust and pressure data during slag-induced "pressure blips" has significantly increased since the original blip studies. This data shows the same behavior as the thrust coefficient deviation predicted by the 1D, 2-phase model. Such a model can set the effective $c^{*}$ and thrust coefficient as a function of slag mass flowed, for better prediction of thrust tailoff and slag mass retained versus time. This in turn will result in better trajectory and vehicle performance data for the Space Launch System or other programs utilizing aluminized boost motors with the propensity to collect slag.

\section{Nomenclature}

\section{English:}

A Area, cross-sectional

$B, C \quad$ Transformation functions

$C_{f} \quad$ Thrust coefficient

$C p$

$c^{*}$

$f_{C p}$

$F$

FoP

Isp

K

$L$

$M$

$m, \mathfrak{w}, m d o t$

$P$

$\operatorname{Pr}$

${ }^{\circ} R$

$R$

$T$

u

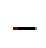

$*$

\section{Specific heat at constant pressure}

Characteristic velocity

Condensed to gas specific heats ratio

Thrust

Thrust to pressure ratio

Specific Impulse

Velocity lag fraction

Thermal lag fraction

Mach number of gas

Mass flow rate

Pressure

Prandtl number

Degrees Rankine

Gas constant

Temperature

Velocity

\section{Overmarks:}

Denotes mixture-based parameter

Superscripts:

Throat condition

\section{Greek:}

$\alpha \quad$ Condensed to gas mass flow rate ratio

$\gamma \quad$ Ratio of specific heats of gas

$\varepsilon \quad$ Expansion ratio

$\rho \quad$ Density

$\begin{array}{ll} & \text { Denotes mixture-based parameter } \\ * & \quad \text { Superscripts: } \\ \text { Throat condition }\end{array}$

\section{Subscripts:}

$0, \mathrm{c}$ Chamber condition

e Exit condition

g Gas

max Theoretical maximum

I, $\mathrm{p} \quad$ Liquid or particle phase (condensed)

\footnotetext{
'Sambamurthi, J. K., A. Alvarado and E. C. Mathias, "Correlation of Slag Expulsion with Ballistic Anomalies in Shuttle Solid Rocket Motors," Journal of Propulsion and Power, Vol. 12, No. 4, July-August 1996, pp. 625-631.
} 
ii Hopson, C., "Space Shuttle Solid Rocket Motor Slag Expulsion Mechanisms," $31^{\text {st }}$ Joint Propulsion Conference, AIAA 95-2725, San Diego, 10-12 July 1995.

iii Martin, C., "Shuttle STS-54 Pressure Perturbation Investigation," 31 $1^{\text {st }}$ Joint Propulsion Conference, AIAA 95-2882, San Diego, 10-12 July 1995.

iv Whitesides, R. H., D. C. Purinton, J. E. Hengel and S. E. Skelley, "Effects of Slag Ejection on Solid Rocket Motor Performance," 31 $1^{\text {st }}$ Joint Propulsion Conference, AIAA 95-2724, San Diego, 10-12 July 1995.

'Dotson, K. W., J. W. Murdock and D. K. Kamimoto, "Launch Vehicle Dynamic and Control Effects from Solid Rocket Motor Slag Ejection," Journal of Propulsion and Power, Vol. 15, No. 3, May-June 1999, pp. 468-475.

vi Kliegel, J. R., One Dimensional Flow of a Gas Particle System, TR-59-0000-00746, Space Technology Laboratories, Inc., Los Angeles, 1959.

vii Dunn, S. S., D. E. Coats and J. C. French, SPP'04 Standard Stability Prediction Method for Solid Rocket Motors Axial Mode Computer Program User's Manual, Software \& Engineering Associates, Inc., Carson City, 2005, pg. 2-2.

viii Cox, D. M., STS-54 Pressure 'Blip' Assessment Report Prepared in Support of UUEC Investigation Effort, Thiokol Report, 15 March 1993. 


\title{
One-dimensional, Two-phase Flow Modeling Toward Interpreting Motor Slag Expulsion Phenomena
}

\author{
Timothy P. Kibbey ${ }^{1}$ \\ Jacobs Technology, Jacobs ESTS Group, Huntsville, AL 35806
}

\begin{abstract}
Aluminum oxide slag accumulation and expulsion can be an important player in various solid rocket motor phenomena. Analysis of un-commanded side accelerations near the end of burn, pressure deviations during steady burn and tailoff, and flight performance knockdown observations all would benefit from improved understanding of slag behavior and its interaction with the gas flow in the motor. One-dimensional, two-phase compressible flow calculations yield an analytical result for nozzle flow under certain assumptions, which can be modified to link motor pressure, thrust, and mass flow rate of gas and slag. An unknown parameter is correlated to airflow testing with water injection. By comparing to thrust and pressure observations on several static test motors, and a slag velocity observation on one flight motor, the model appears to predict the proper form of dependency on slag-to-gas mass flow rate ratio, with an adjustment required for thrust only.
\end{abstract}

\section{Nomenclature}

$A=$ Area, cross-sectional

$B, C=$ Transformation functions

$C_{f}=$ Thrust coefficient

$C p=$ Specific heat at constant pressure

$c^{*}=$ Characteristic velocity

$d t \quad=$ Change in time

$F \quad=$ Thrust

$f_{C p}=$ Condensed-to-gas specific heats ratio

$\left.\Delta F\right|_{r}=$ Thrust enhancement factor

$I s p=$ Specific Impulse

$K=$ Velocity lag fraction

$K s \quad=$ Slag Ballistics Model density factor

$L=$ Thermal lag fraction

$M=$ Mach number

$\dot{m}=$ Mass flow rate

$n=$ Burn rate exponent

$P \quad=$ Pressure

$P r=$ Prandtl number

$R=$ Gas constant

$R_{F o P}=$ Ratio of thrust-over-pressure ratio of data to thrust-over-pressure ratio of scaled nominal

$T=$ Temperature

$u \quad=$ Velocity

$\alpha=$ Condensed-to-gas mass flow rate ratio

$\gamma \quad=$ Ratio of specific heats

$\varepsilon=$ Expansion ratio

$\rho \quad=$ Density

\footnotetext{
${ }^{1}$ Senior Engineer, Propulsion Engineering, MSFC ER-51.
} 
Subscripts and Symbols

$\begin{array}{ll}c & =\text { Chamber condition } \\ e & =\text { Exit condition } \\ g & =\text { Gas } \\ \text { init } & =\text { Initial } \\ p & =\text { Particle } \\ \text { nomsc } & =\text { Nominal trace scaled to data } \\ -\quad & =\text { Denotes two-phase mixture-based parameter } \\ * \quad & =\text { Throat condition }\end{array}$

\section{Introduction}

A luminum oxide slag accumulation and expulsion was previously ${ }^{1,2,3}$ shown to be a player in various solid rocket motor phenomena, including the Space Shuttle's Reusable Solid Rocket Motor (RSRM) pressure perturbation, or "blip," and phantom moment. In the latter case, such un-commanded side accelerations near the end of burn have also been identified in several other motor systems. However, efforts to estimate the mass expelled during a given event have come up short. Either bulk calculations are performed without enough physics present, or multiphase, multidimensional Computational Fluid Dynamic analyses are performed that give a snapshot but are difficult to expand to enough generality.

One-dimensional (1D), two-phase compressible flow calculations yield an analytical result for nozzle flow under certain assumptions. This can be carried further to relate the bulk motor parameters pressure, thrust and mass flow rate under the different exhaust conditions driven by the addition of condensed phase mass flow. An unknown parameter is correlated to airflow testing with water injection ${ }^{4}$. Also, comparison is made to full-scale static test motor data, where thrust and pressure changes are known, and similar behavior is shown.

The end goal is to be able to include the accumulation and flow of slag in internal ballistics predictions. This will allow better prediction of the tailoff, when much slag is ejected, and of mass retained versus time, believed to be a contributor to the widely-observed "thrust knockdown" parameter. While a previous model required an integration of a slag bit's velocity as it travels through the nozzle, the present model results in discharge and thrust parameters as explicit functions of the slag mass flow ratio, enabling direct inclusion into existing ballistics prediction models.

\section{Two-Phase Flow Model}

Previous analyses ${ }^{4,5}$ have treated the slag flow as a monolithic inert mass partially blocking the nozzle as it flows overboard. These estimate the momentum of the inert mass, but do not account for the momentum reduction of the gas required to accelerate the inert mass, thus violating conservation of momentum. Since the pooled slag is thought by these authors to be a liquid-gas slurry, and to strongly interact with the upstream gas flow, perhaps a more diffuse model of interaction is appropriate. This is especially true if the slag does attain significant momentum when flowing through the nozzle, which would be debited from the gas flow.

A one-dimensional two-phase compressible flow model was derived for solid rocket motor nozzle flows by Kliegel ${ }^{6}$. The analysis assumes full momentum and convective thermal coupling between phases, but no particle-toparticle interaction; thus particles can be either liquid or solid. It also assumes an ideal, inviscid gas, except for drag on the particles, of constant properties, and no chemical reactions. An analytic derivation is possible assuming spherical particles in Stokes flow and a constant fractional lag, $K$, meaning as the flow accelerates through the nozzle, the particle's velocity is always the same fraction of the gas velocity at the same location. A discussion of how valid an approximation this might be of actual non-uniform behavior is reserved for a later section.

\section{A. Derivation}

Kliegel's solution is presented in Eqs. (1) through (7), showing the same form as isentropic flow, but with modified Mach number $(\bar{M})$ and specific heats ratio $(\bar{\gamma})$. This includes the following parameter definitions:

$$
\begin{gathered}
B=\frac{1+\alpha K^{2}}{1+\alpha f_{C p} L} \\
C=1+\alpha\left[K(\gamma(1-K)+K)+(\gamma-1) f_{C p} L B\right] \\
\bar{M}=\sqrt{C} M
\end{gathered}
$$




$$
\begin{gathered}
\bar{\gamma}-1=(\gamma-1) \frac{B}{C} \\
\frac{1-L}{L}=3 \operatorname{Pr} f_{C p} \frac{1-K}{K} \\
h(\bar{M})=1+\frac{\bar{\gamma}-1}{2} \bar{M}^{2}, \frac{T_{c}}{T_{g}}=h(\bar{M}), \frac{P_{c}}{P}=(h(\bar{M}))^{\frac{\bar{\gamma}}{\bar{\gamma}-1}}, \frac{\rho_{c}}{\rho_{g}}=(h(\bar{M}))^{\frac{1}{\bar{\gamma}-1}} \\
u_{g}{ }^{2}=\frac{2 C p_{g} T_{c}}{B}\left(1-\frac{T_{g}}{T_{c}}\right)
\end{gathered}
$$

Note that, when $K=L=1$, this reduces to the heavy gas assumption ${ }^{7}$. One result is that the transformed mixture Mach number, $\bar{M}$, is 1 at the geometric throat, while the gas Mach number, $M$, is less than 1 . This applies also to standard RSRM flow, where the heavy gas reduction is typically used for ballistic analysis. Herein, when referring to the "RSRM gas" these heavy gas properties will be used. A future analysis could attempt to derive the equations with multiple particle sizes subject to multiple lag ratios representing smoke, larger particles in the flow, and pooled slag ejecta to see if higher fidelity can be achieved.

A change in characteristic velocity $\left(c^{*}\right)$ due to the addition of condensed flow can be derived, which will tie mass flow rate to chamber pressure. Then, a change in thrust coefficient $\left(C_{f}\right)$ can be derived, tying in thrust and Specific Impulse (Isp).

\section{B. Connecting Mass Flow to Pressure, $c^{*}$}

Consider mass flow rate at the nozzle throat, invoking the ideal equation of state for the gas phase and the above equations with continuity, resulting in Eq. (8).

$$
\dot{m}=\dot{m}_{g}+\dot{m}_{p}=\dot{m}_{g}(1+\alpha)=\rho_{g}^{*} A^{*} u_{g}^{*}(1+\alpha)=\frac{P^{*}}{R_{g} T_{g}^{*}} A^{*} \sqrt{\frac{\gamma R_{g} T_{g}^{*}}{C}}(1+\alpha)
$$

Substituting Eq. (6) puts Eq. (9) in terms of chamber conditions.

$$
\overline{c^{*}}=\frac{1}{(1+\alpha)} \sqrt{\frac{C \operatorname{Cg}_{g} T_{C}}{\gamma}\left(\frac{\bar{\gamma}+1}{2}\right)^{\bar{\gamma}+1}}
$$

Note the appearance of both gas $\gamma$ and mixture $\bar{\gamma}$ in Eq. (9), and its similarity to the standard gas $c^{*}$ with the addition of $C$ and $(1+\alpha)$.

The goal is to show a chamber pressure increase due to addition of condensed flow. Given condition 1 with only gas flow and condition 2 with the same gas flow rate plus condensed flow, the ratio of pressure 2 to pressure 1 is given in Eq. (10). This is the appropriate form to apply to the air/water flow data below.

$$
\frac{P_{c 2}}{P_{c 1}}=\frac{\left(\dot{m} c^{*} / A^{*}\right)_{2}}{\left(\dot{m} c^{*} / A^{*}\right)_{1}}=(1+\alpha) \frac{\overline{c^{*}}}{c^{*}}=\sqrt{C \frac{\left(\frac{\bar{\gamma}+1}{2}\right)^{\frac{\bar{\gamma}+1}{\gamma-1}}}{\left(\frac{\gamma+1}{2}\right)^{\frac{\gamma+1}{\gamma-1}}}}
$$

\section{Connecting Thrust to Pressure, $C_{f}$}

The steady-state momentum balance gives the thrust in Eq. (11), here assuming vacuum conditions.

$$
F=\dot{m}_{g} u_{g e}+\dot{m}_{p} u_{p e}+P_{e} A_{e}=\dot{m}_{g} u_{g e}(1+\alpha K)+P_{e} A_{e}
$$

With $\dot{m}_{g}=\frac{\dot{m}}{(1+\alpha)}$, Eqs. (8) and (9) substitute $\dot{m}_{g}$, and Eq. (7) substitutes gas velocity to give Eq. (12). 


$$
\overline{C_{f}}=(1+\alpha K) \sqrt{\frac{2 \gamma^{2}}{C B(\gamma-1)}\left(\frac{2}{\bar{\gamma}+1}\right)^{\bar{\gamma}+1} \frac{(\bar{\gamma}-1) \bar{M}^{2}}{2+(\bar{\gamma}-1) \bar{M}^{2}}}+\frac{P_{e}}{P_{C}} \varepsilon
$$

Now the thrust ratio can be solved, with Eq. (13) again showing the similarity in form to the standard gas $C_{f}$.

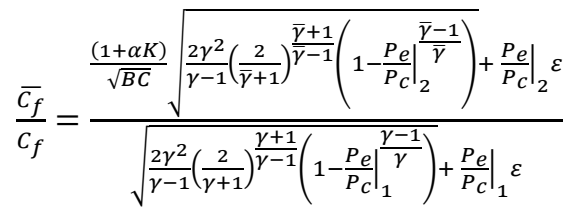
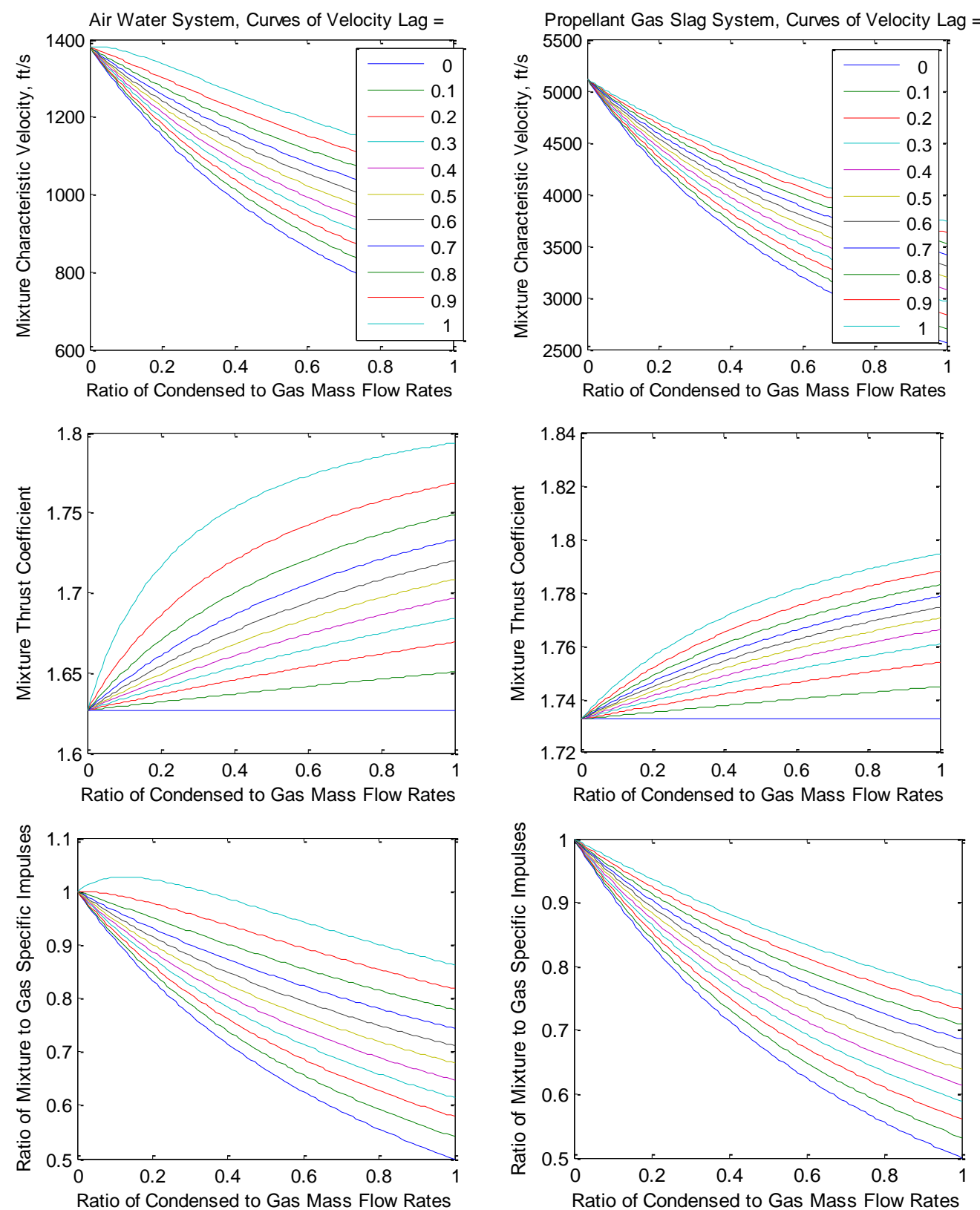

a)

b)

Figure 1. Two-Phase Flow Model Results for a) Air Water, and b) Gas Slag Properties

American Institute of Aeronautics and Astronautics 
Table 1. Properties

\begin{tabular}{|l|c|c|}
\hline & Air/Water & RSRM Gas/Slag \\
\hline$\gamma$ & 1.4 & 1.155 \\
\hline $\mathrm{R},[\mathrm{ftlb}] /\left[\mathrm{lb}^{\circ} \mathrm{R}\right]$ & 53.33 & 54.54 \\
\hline $\mathrm{T}_{\mathrm{c}},{ }^{\circ} \mathrm{R}$ & 520 & 6092 \\
\hline $\mathrm{Pr}$ & 0.7 & 0.8 \\
\hline$f_{C p}$ & 4.18 & 1.5 \\
\hline$\varepsilon$ & 7.72 & 7.72 \\
\hline
\end{tabular}
Center. Two was with water injected behind the nozzle cavity, representative of where slag would collect in a solid rocket motor; this is the Main Data on Fig. 2, where Eq. (10)'s ratio of pressure with and without condensed flow is plotted against $\alpha$. The "Precursor Data" for the other tests is included for comparison.

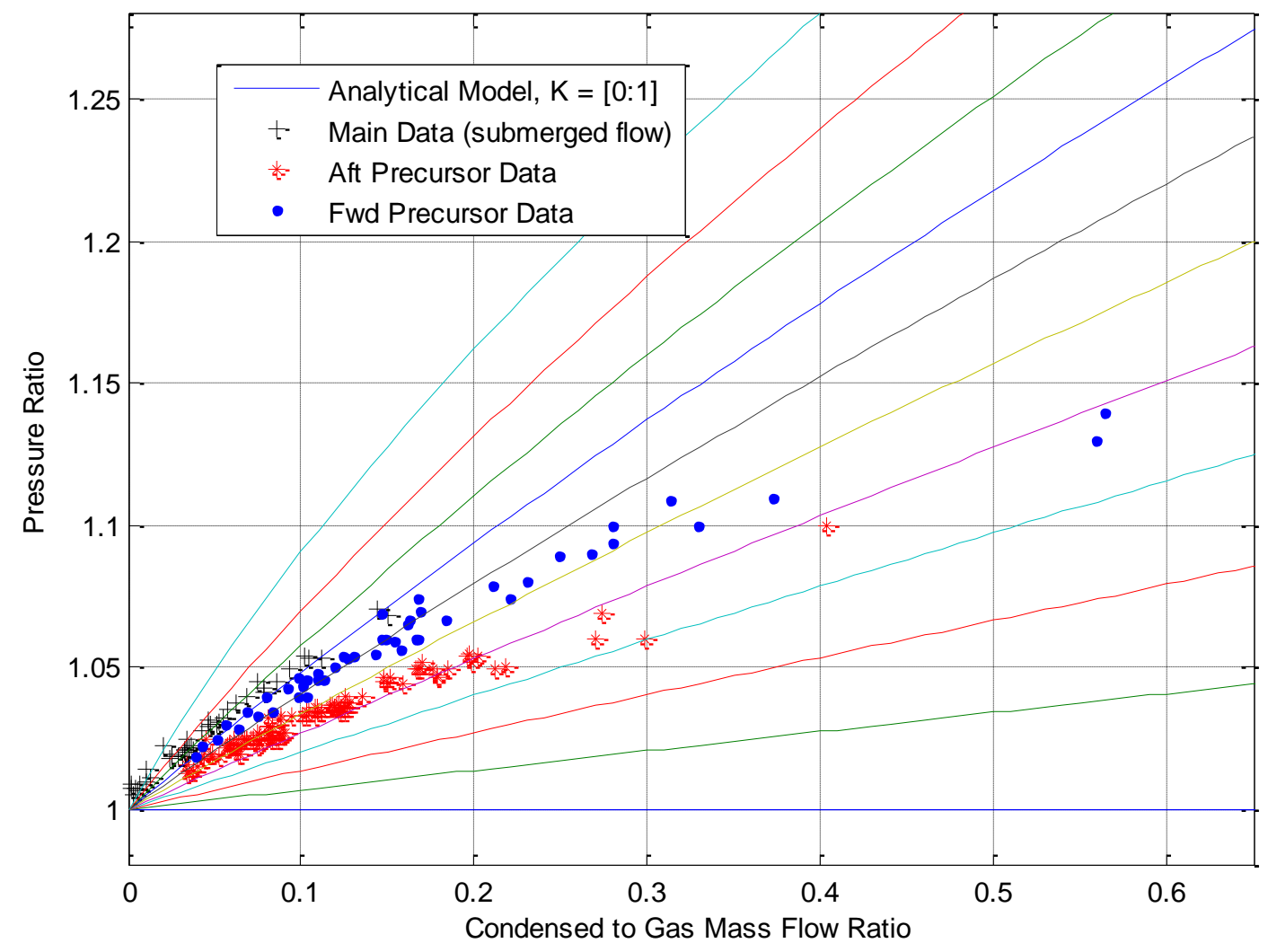

Figure 2. Water Injection Testing Correlated to Analysis

The two sets of "Precursor Data" injected water through a jet at the centerline of the flow chamber. "Aft" used an injection point located just upstream of the nozzle while "Fwd" was several feet upstream. Compared to the "Main Data," where water seeped in through 12 openings, the precursor injections added significant momentum to the flow. For this reason it is not surprising that the aft data shows much lower pressure rise for a given flow rate.

American Institute of Aeronautics and Astronautics 


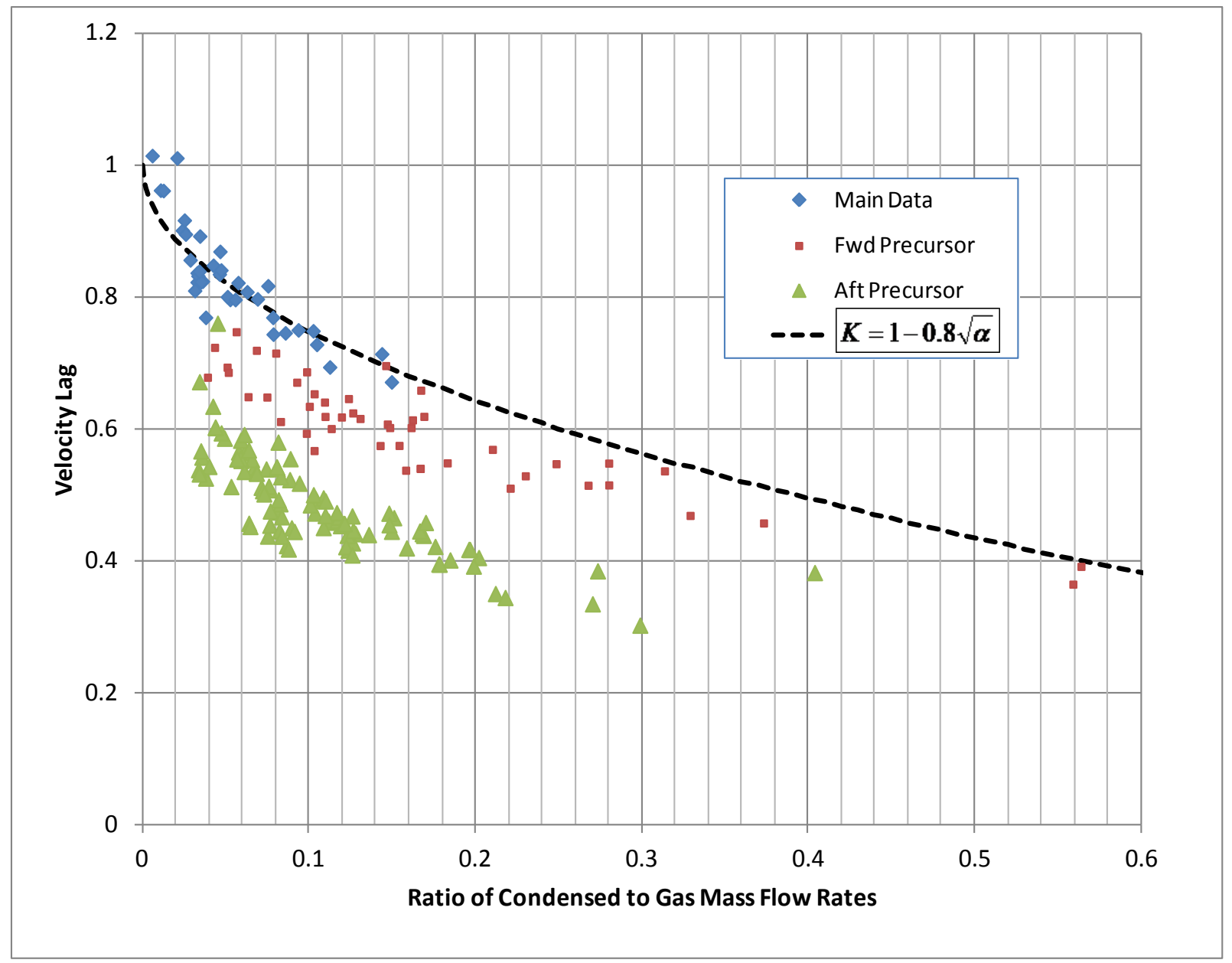

Figure 3. Determination of Velocity Lag, $K$, from Air-Water Data

Though the forward data had the same injector and nozzle, the flow likely had time to lose some of the water momentum to the wall by friction, so it approached the main data. In addition, each precursor stream likely resulted in a different particle size through the nozzle than the main data.

The data can be turned around to fit a curve relating $K$ to $\alpha$. This is given in Fig. 3, where the precursor data is also shown for comparison. Clearly for full confidence, the Main Data curve fit should only be used within the data range. $K$ as a function of $\alpha$ sums up all the properties related to particle drag, thus simply defining the interaction without needing to know several particle and flow characteristics.

\section{B. Full-Scale Motor Static Test Data}

At the time of the focused RSRM pressure blip studies, Martin ${ }^{3}$ observed from the data of one static test motor that the thrust-to-pressure ratio (FoP) increased during a blip, and that the thrust rise slightly led the pressure rise. This is consistent with the model presented herein, where the FoP increases due to the thrust coefficient increase. The database of thrust behavior during pressure blips has significantly increased since then. For the current effort, a consistent data processing and analysis method was developed to examine 25 blips from the 60-80 s timeframe on 6 motors.

\section{Blip Data Analysis}

The measured thrust, converted to vacuum, and pressure data are smoothed and the nominal trace is scaled to match the region near the pressure blip of interest. During the time of many blips, both vertical and horizontal vectoring is important, and the effect of vectoring is on the order of the increase in FoP, so it is very important to account for both transverse axes as well as axial thrust. By examining these two traces, a blip start time is selected 
and the nominal is scaled again to match the pressure of the blip start. The ratio of actual to no-blip FoP, Eq. (14), can then be compared to $\frac{\overline{C_{f}}}{C_{f}}$ in the two-phase flow model.

$$
R_{\text {FoP }}(t)=\frac{F / F_{\text {no blip }}}{P / P_{\text {no blip }}}
$$

$\mathrm{P}_{\text {no blip }}$ is just the interpolated scaled nominal versus time, but $\mathrm{F}_{\text {no blip }}$ must be adjusted from the interpolated scaled nominal (interpolated $F_{\text {nomsc }}$ ) to correct for motor variation of FoP that is unrelated to blip. This is done by computing the ratio of data FoP to nominal FoP and averaging the half second prior to the blip to establish a baseline FoP ratio, $R_{F o P}$ init. An example of this is shown in Fig. 4 . There is a clear quasi-steady FoP match prior to the blip start, and a clear rise in FoP during the blip, but the initial value is not 1.

To properly finish Eq. (14), compute the motor-specific no-blip thrust, Eq. (15).

$$
F_{\text {no blip }}=\left(\text { interpolated } F_{\text {nomsc }}\right) * R_{\text {FoP init }}
$$

The above results provide a time-based point-by-point comparison of the pressures and thrusts during a blip. Two simplifications make this an imperfect, but adequate, method, as will be shown below. First, the transient behavior that results in the pressure slightly lagging the thrust is not modeled, but focusing on the peak of the blip allows a quasisteady assumption, as will be demonstrated below. Second, due to the pressure increase, the burn rate will increase. The best comparison is to match each data point with the no-blip point at the same burn distance, rather than same time. However, a conservative analysis shows such a slight difference in time and pressure that it doesn't matter: if a pressure difference of 10 psi were sustained for $1 \mathrm{~s}$, the ratio of burn rates and times to get to the

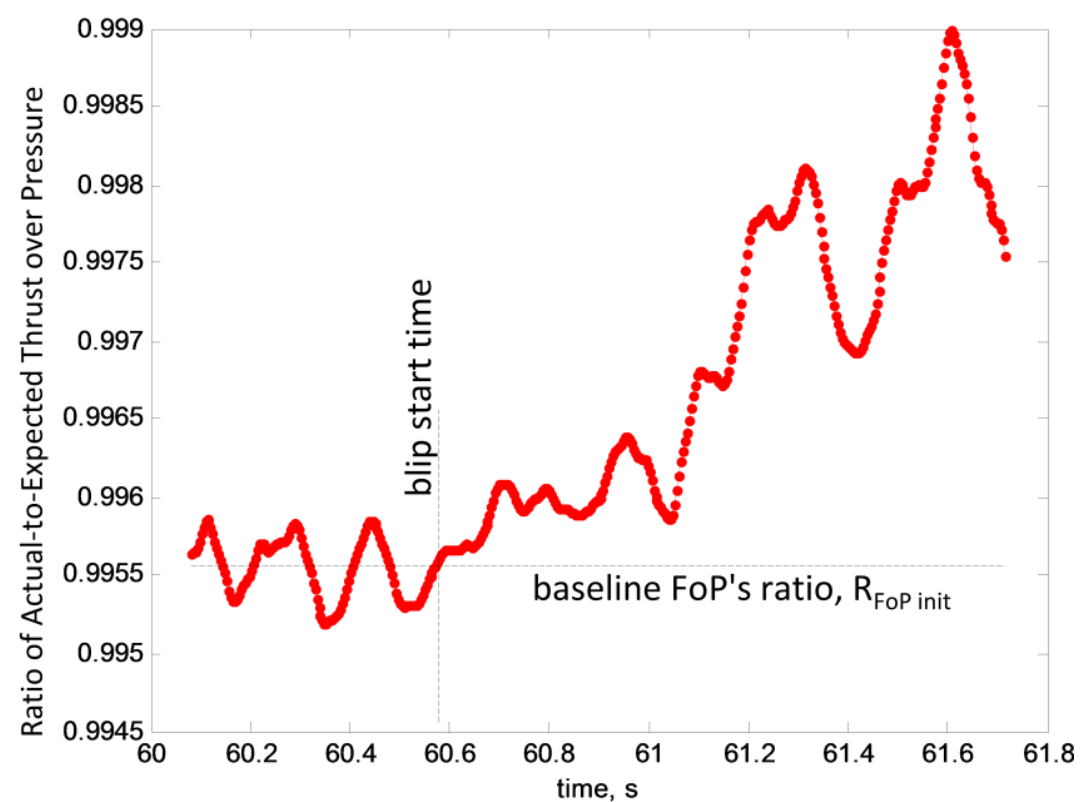

Figure 4. Flight Support Motor (FSM-) 13 FoP Baselining Example

same burn distance would be as in Eq. (16). Here, $n=0.32$ is the burn rate exponent. For pressures around those of these blips, the change in time $d t$ over $1 \mathrm{~s}$ of data is only $0.005 \mathrm{~s}$ of nominal, which results in less than $0.02 \mathrm{psi}$ difference.

$$
\frac{\dot{r}_{\text {blip }}}{\dot{r}_{\text {no blip }}}=\frac{1 \mathrm{~s}+d t}{1 \mathrm{~s}}=\left(\frac{P_{\text {blip }}}{P_{\text {no blip }}}\right)^{n}=1.005
$$

One of the large blips on FSM-7 will be used as an example of the analysis. Figure 5 shows this blip with the appropriate scaling of the nominal. The above equations result in Fig. 6, with the ratio of blip to no-blip FoP's on the left, and the thrust rise plotted against the pressure rise on the right. In both these, the hysteresis is evidence of the thrust rise leading the pressure rise. The thrust increase rolls over approaching the quasi-steady peak of the blip, then the thrust decrease again leads on the way down. It is expected that if the fully transient behavior were modeled, the rising and falling portions of the curve would converge toward the central slope captured by the peak value. A linear fitting scheme of the rising portion was attempted to determine $R_{F o P}$ as a function of $P / P_{n o}$ blip. However, this 

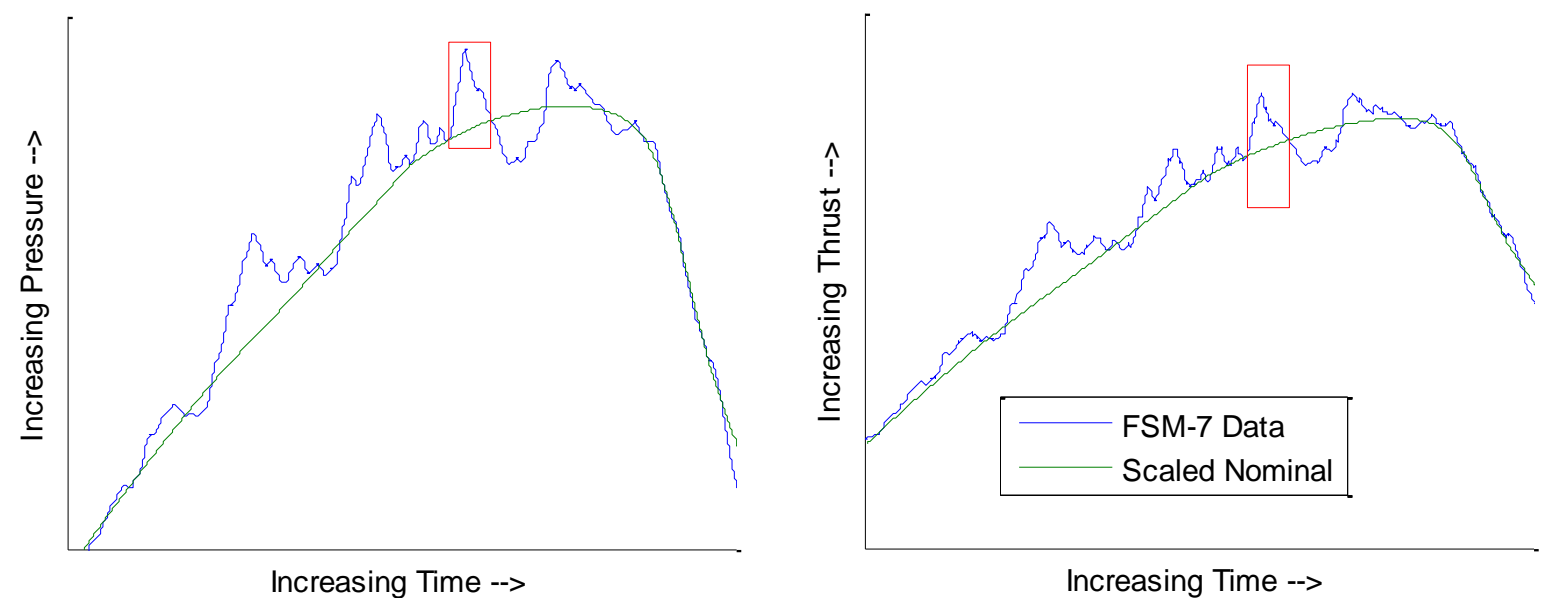

Figure 5. FSM-7 Data with Nominal Scaled for the Blip at $70 \mathrm{~s}$
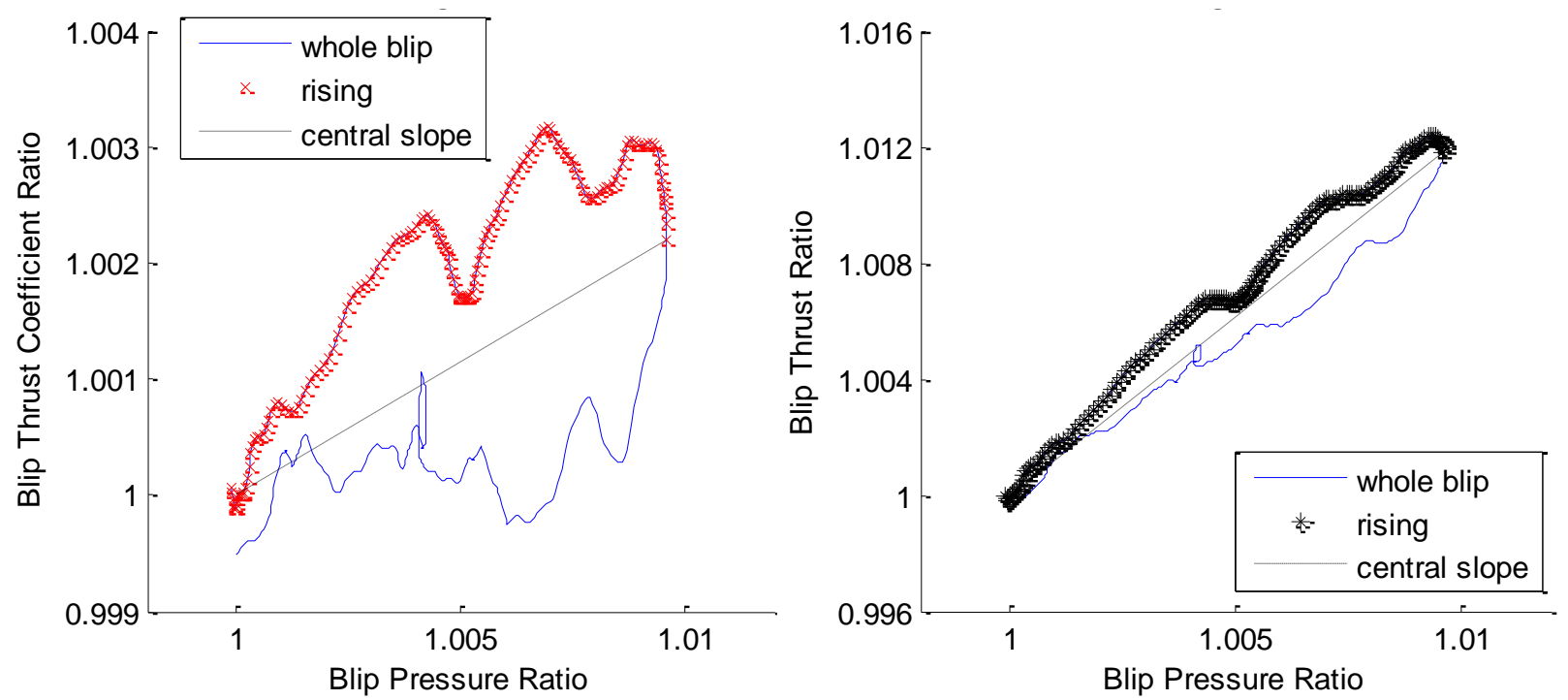

Figure 6. Processing of FSM-7's 70 s Blip

suffered the drawbacks of ignoring the synch-up of $(1,1)$ that drew on the previous half-second of data and of strong response to transient rise and spurious variation. This resulted in high data scatter when all the blips were compiled.

Having observed all the blips in this manner, the same general behavior was exhibited. And though much variation was seen in the rising portion, the peak region proved quite consistent. So, to compile the effect, the $R_{F o P}$ at peak $P / P_{\text {no blip }}$ was selected as the $\frac{\overline{C_{f}}}{C_{f}}$ for that pressure ratio. Note that peak of blip is defined here as the maximum of the ratio $P / P_{\text {no blip }}$, not the local maximum pressure.

\section{Blip Thrust Coefficient Results}

The results of this analysis are presented for the 25 blips in Fig. 7. The left hand figure shows a discernible correlation, perhaps locally linear. However, further examination of the data suggests that it could be fit better by treating as two populations, shown tightly correlated in the right hand figure as high- and low-slope groups.

Figure 8 examines the distribution of each point's individual $\frac{\overline{C_{f}}}{C_{f}}$ slope, adding further credibility to the thought of 2 populations; note the histogram's double-hump nature, and the gap in slopes around 0.18 . More work would be 


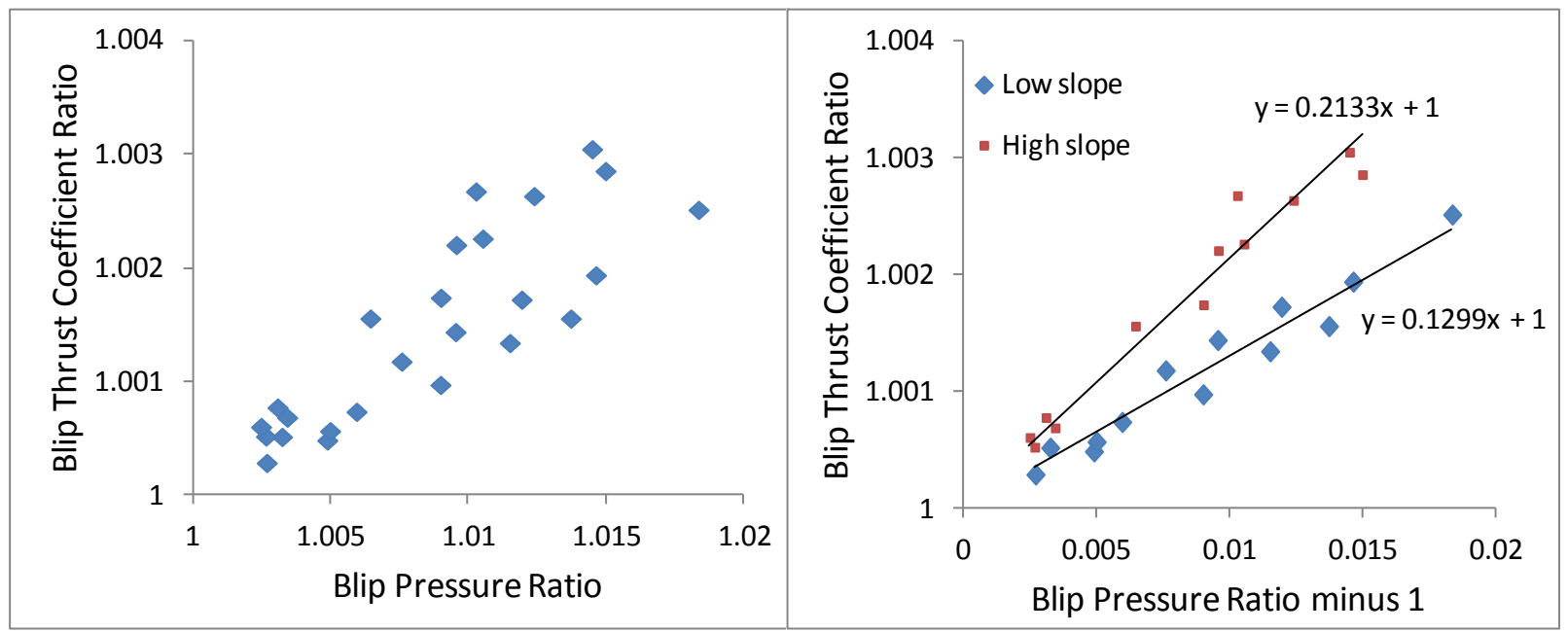

Figure 7. RSRM Blip FoP Study Results

required to identify a cause for the difference between the two types. It may depend upon the proximity of blips to each other or the duration of the blip, perhaps similar to Hopson's ${ }^{2}$ observation about "slosh" and "spill family" events on flight motors.

Now these data can be compared to the prediction of the two-phase flow model, using the Table 1 properties for the RSRM gas/slag system. They are plotted together in Fig. 9, where the model exhibits the same trend shape, but at a lower level than the data. The model can be adjusted to overlap the data either by scaling with a constant, exemplified in the figure, or by adjusting the gas properties. The latter course will be discussed later. Given the simplifying assumptions that were made in model development, one should not be surprised to find that a scaling constant is required to match the data. Rather, one is pleased to find the correct form of dependence on the flow parameters. Indeed, it is found elsewhere in

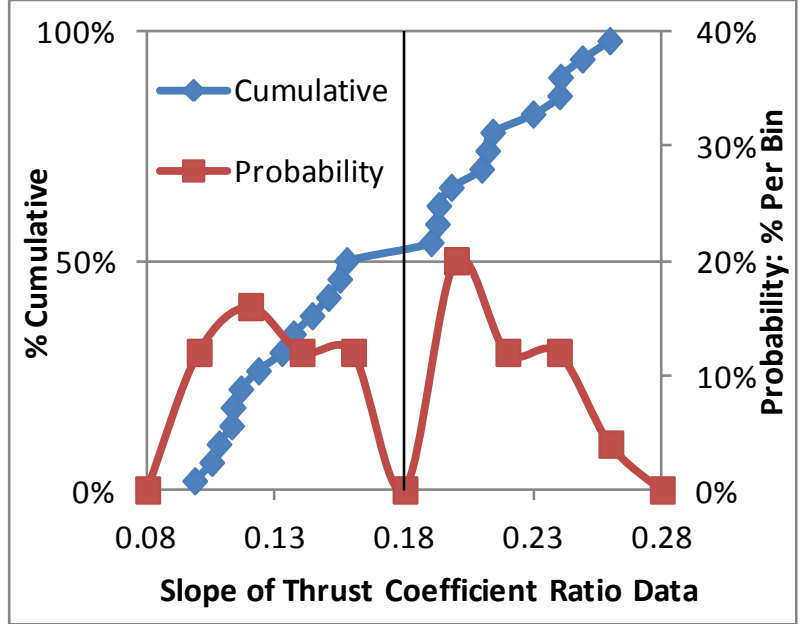

Figure 8. Histogram and Cumulative Distribution of Slopes fluid flow problems that a simplified analysis yields the correct trends, with only coefficients requiring adjustment to match data, momentum integral calculations of a flat plate boundary layer ${ }^{8}$ being one example. Specific reasons for the differences will be discussed below.

An important observation needs to be made here: the property-adjustment method shows that the mass flow result is insensitive to adjusting to match the thrust data. This is not surprising given Fig. 1, where the $c^{*}$ function of the flow parameters showed a much tighter band than the $C_{f}$ function. So the computations of slag mass flow rate versus pressure blip level shown below need not be adjusted based on this thrust coefficient data.

Note that the range of the model for different $K$ values is more compressed here plotted against $P / P_{n o}$ blip than when plotted against $\alpha$. One result of this is that whether with scaling or property adjustment to match data, the $K(\alpha)$ correlation is neither confirmed nor denied by the data, because the data noise is as large as the model range.

\section{Full-Scale Motor Flight Data}

One datapoint was found for estimating the velocity of ejected slag. On the Ares I-X test flight of a single RSRM, Doppler radar showed a distinct slag event at $118 \mathrm{~s}$ with a discernible velocity difference between the motor and the slag'. Using similar triangles and the vehicle velocity vector from the Best Estimated Trajectory, the slag speed relative to the motor was determined to be $1590 \mathrm{ft} / \mathrm{s}$. This is $20 \%$ of the gas-only exit velocity, suggesting velocity lag $K=0.2$ is a good first estimate. While this is significantly lower than the estimates for blips earlier in burn, there is likely a significantly higher $\alpha$ flowing at this time, which the airflow data and the model suggest 


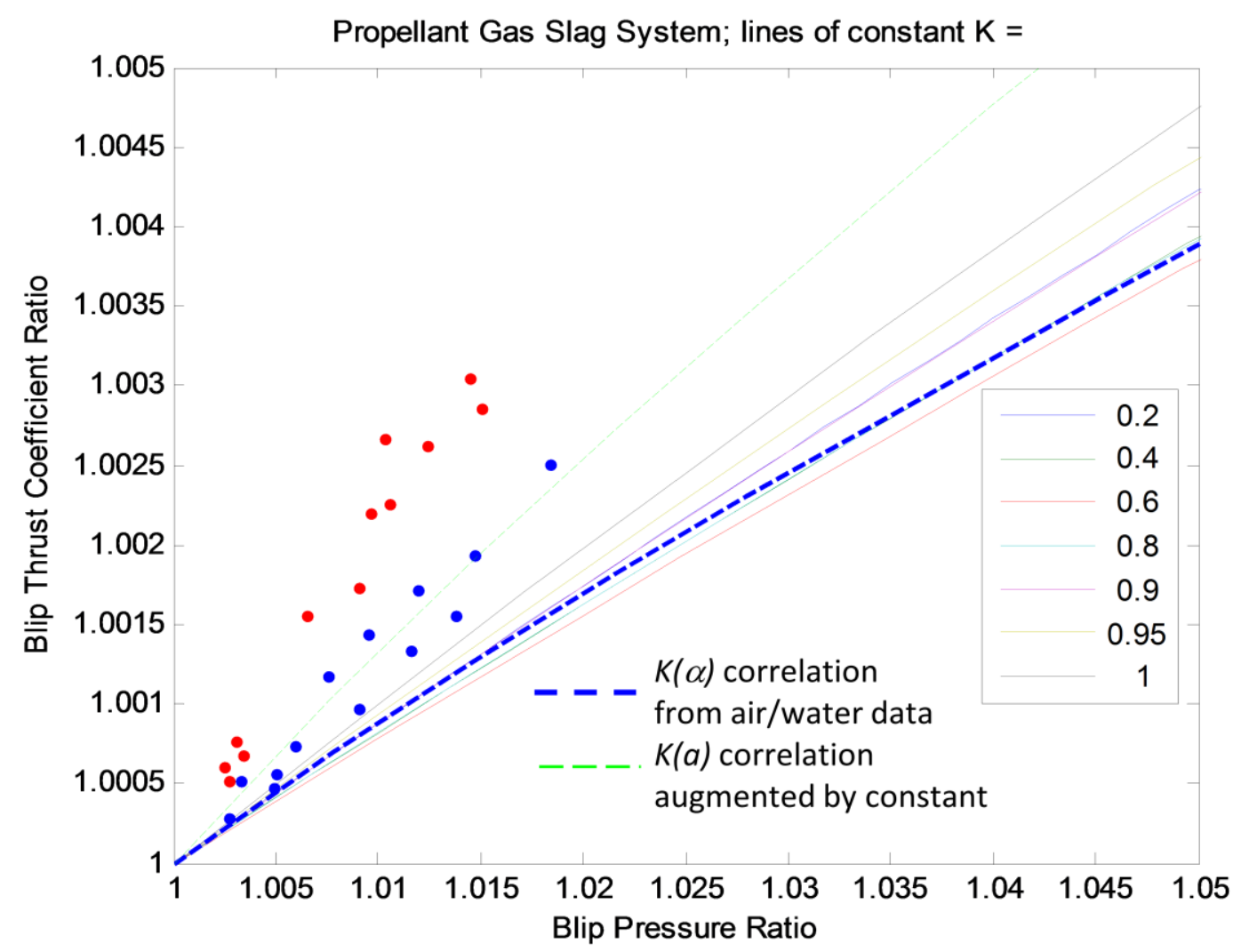

Figure 9. Present Model Showing a Trend Shape Similar to RSRM Blip FoP Data but Different Magnitude

would result in a lower $K$. The correlation of Fig. 3 gives $K=0.2$ at $\alpha=1$. Updating the flow properties to include the second phase reduces the gas speed, and the answer converges to $K=0.22$ and $\alpha=0.95$. At first thought this $\alpha$ may seem quite out of bounds given the low flow ratios earlier in burn, but the pressure here is only 48 psi, so this high $\alpha$ results in only about twice the slag mass flow rate of the regular earlier pressure blips. This result is reasonable for the following reasons: Static test radiometer data shows the highest, most sustained signatures during the latter tailoff, less than $150 \mathrm{psi}$. The higher increase in radiometer reading signals a higher impact of slag in the plume, though a quantitative link between radiometer heat flux and slag mass flow has not been demonstrated. Pressure blips cannot be seen at this level because the depressurization is so steep, but an examination of flight and static test data shows a deviation from a standard ballistics prediction starting around 150 psi. This effect is more pronounced for flight than static test, but present in both cases. This suggests that thousands of pounds of slag have accumulated and are expelled in the last 5-10 s, reducing the slag retained to the typical post-fire value of 1000-3000 $\mathrm{lbm}$.

In order to further evaluate this assessment, an examination of any other slag radar indications from Shuttle launches or other launch vehicles would be useful.

\section{Comparison to Previous "Slag Ballistics Model"}

The prevailing model in the literature for quantifying slag mass flowed and thrust effects is the "Slag Ballistics Model (SBM)," presented with the air/water flow study used herein ${ }^{4}$. That model computed an effective throat area required to raise the pressure the amount measured, with the $c^{*}$ unaffected. Then drag calculations led to slag mass flow rate and velocity, without affecting the gas flow that dragged the condensed phase. These calculations included a density-muliplying factor, $K s$, meant to increase the effective volume of the slag to represent that it probably comes out of the slag pool as a mixture of liquid and gasses. Finally, thrust is computed by adding the slag jet's mass flow times velocity to the gas thrust, based on the effective throat area with $C_{f}$ unaffected.

The primary thrust effect metric in the SBM is the "Thrust Enhancement Factor," $\left.\Delta F\right|_{r}$, shown in Eq. (17), where $R_{F o P}$ and $P$ are calculated at the blip peak, and $P_{I}$ is the pressure prior to the blip. An approximation of $\left.\Delta F\right|_{r}$ 


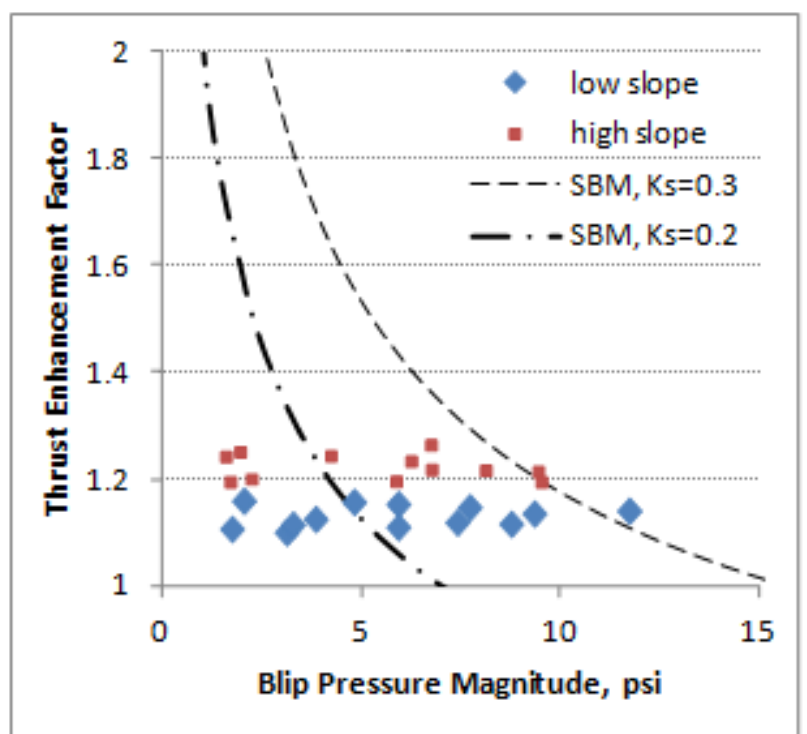

a)

Figure 10. Previous Slag Ballistics Model Showing its Inability to Capture the Trend Shape of RSRM Blip FoP Data: a) Thrust Enhancement Factor and b) Ratio of Thrust Coefficients

rederived from the SBM equations will be used below to compare SBM results to the pressure blip thrust data. The approximation was checked against the published SBM plots to ensure it exhibits similar behavior.

$$
\left.\Delta F\right|_{r}=\frac{R_{F o P} * P-P_{1}}{P-P_{1}}
$$

Figure 10a plots two such curves along with the thrust enhancement factor calculated for all the static test blip data. The density-muliplying factor for each curve required tweaking to match different data: $K s=0.2$ matched the known air/water mass flow rate data without known thrust; $K s=0.3$ curve matched the Qualification Motor 1 static test known thrust data point without known mass flow rate. Now that more than one static test motor thrust and pressure data point is available, the inadequacy of the SBM is apparent. Furthermore, convert the SBM curves to $\mathrm{R}_{\mathrm{FoP}}$ by rearranging Eq. (17), shown with the motor data in Fig. 10b. Absent from the data is any indication of these ratios dropping below 1, as in the SBM when throat blockage overwhelms slag momentum at increased pressure deltas. These comparisons are not exact because the SBM references the initial pressure while the blip analysis used the nominal as it changed during the blip, but adjusting the reference would merely reduce the slope of the data a bit, not change its shape. So the conclusion is clear: the SBM is of different character than the blip thrust and pressure data, while back in Fig. 9 it was seen that the two-phase flow model is of the same character as the data.

The SBM slag velocity analysis can also be compared by considering the aforementioned Ares I-X ejection velocity datum. A recalculation with the SBM of the STS-54 blip estimates a velocity lag factor of only 0.1 through the throat. If such an event causing a $2 \%$ pressure blip only manages to accelerate the slag to $10 \%$ of the gas speed according to the SBM, is it unlikely the SBM would predict the higher $20-22 \%$ value measured on Ares I-X for what is likely a significantly larger slag event at low gas flow near the end of burn.

\section{E. Further Discussion of Model Validity}

One concern regarding the usefulness of this model is that the condensed phase is known to often be localized to one zone of the nozzle, whereas this technique computes it in a 1D manner. This is seen by plume radiometer and imagery data during blips and by blip-caused moments late in burn. The impact of the condensed phase is to change the expansion parameters of the flow, primarily reducing flow acceleration and pressure decrease while it expands through the nozzle. Thus, a flow laden with particles would have a higher pressure at each axial position than a gasonly flow. If both are present in the same nozzle, this would set up two different pressure regions. While this is feasible to consider in the supersonic flow downstream of the throat, in the converging section there is full communication of pressure across the cross-section. That is, if the condensed flow sector tries to maintain a higher pressure, this information is transmitted by pressure waves across the cross-section, averaging out the pressure in some manner. As the 1D two-phase pressure/mass flow model is consistent with the air/water flow data, it seems to

11

American Institute of Aeronautics and Astronautics 
approximately capture this effect by treating the flow up to the throat as $1 \mathrm{D}$ and diffuse. The more local nature of flow downstream of the throat could be one reason that model adjustment is required to match thrust data.

This is supported by the air-water data further, comparing tests with two different nozzle settings (Fig. 11). Tests with a $4^{\circ}$ cant are believed to have a different local flow distribution of condensed material than the straight nozzle, with the material favoring the lower canted side. But the data shows no impact on the bulk effect of pressure increase, further suggesting that the model's lack of local distinguishing is adequate.

As for the flow downstream of the throat, pressure data is communicated downstream along characteristic lines, such that a localized flow condition has a localized effect. The result on thrust would be higher pressure along the wall near the condensed flow, giving a side thrust and an axial thrust increase. In a fully heavy gas flow with infinitesimal particles, this would be a smooth gradation of pressure. In a more discrete flow, this would manifest as shocks traveling obliquely from each particle to the wall. In either case the net effect of increased pressure at the wall, increased axial thrust, and a side thrust is the same. Given this, one would expect the thrust impact of the 1D two-phase model to fail quantitatively, which it does in raw form. But the qualitative description and the matching of the general characteristics of the model to the full-scale thrust data by showing the proper sensitivity to variables suggest that the model captures the essence of the main effects. In sum, the localized real effects of two-phase flow aft of the throat are one reason that scaling or parameter modification must be used to match the thrust data.

Notwithstanding the discussion about physical averaging of the flow upstream of the throat, an attempt was made to analyze more locally by using a region affected by slag-gas two-phase flow and an unaffected region, but it didn't pan out.

\section{Geometric and Fluid Property Considerations}

The original two-phase flow derivation includes a velocity equation containing the axial coordinate through the nozzle. Tying this in with the other equations defines a certain contour capable of

delivering the constant lag flow that was assumed. Fig. 12 compares this optimal profile to the RSRM nozzle inlet at the same scale. This similar profile suggests that calculations through the throat, such as $\mathrm{c}^{*}$ and thus mass flow rate, will be reasonable. The profile downstream of the throat departs significantly, suggesting $C_{f}$ and thrust could be less accurate than values dependent only on conditions up to the throat. Thus, the nozzle contour aft of the throat is another reason that scaling or parameter modification must be used to match the thrust data.

It is also worth discussing the properties used with the RSRM propellant gas-slag model. Because the two-phase flow formulation assumes constant gas properties, the frozen flow specific heat and the $\gamma$ based on it were used. Remember that this is also for a two-phase mixture, including all the aluminum oxide suspended in the flow as

12

American Institute of Aeronautics and Astronautics 
smoke and small particles assuming heavy gas behavior. In attempting to match the data, I found that a $\gamma$ closer to the single-phase value of the propellant gas-only portion would allow alignment. I am hesitant to adopt this overtly, without a more extensive analysis taking into account flow properties with both small and large particle flow in a coupled sense. Such a $\gamma$ could nevertheless be used as a "pseudo- $\gamma$." With or without a "pseudo-property," the twophase flow model provides the appropriate shape of the data, even though it is very much simplified and missing many details: non-constant gas behavior, interface between true gas and a range of particle sizes, shocks aft of the throat, non-symmetric non-dispersed nature of the flow, particles breaking up through and/or after the throat, threedimensional effects, etc.

\section{Effect of Condensed Phase Throat Area Blockage}

The cross-sectional area occupied by the condensed phase was not included in the model. In previous models, area reduction due to condensed phase cross-section has been viewed as the primary mechanism of pressure perturbation. In the air-water case, assuming a water density of $62 \mathrm{lbm} / \mathrm{ft}^{3}$, the maximum tested $\alpha$ of 0.15 blocks $0.4 \%$ of the throat. This means that about $6 \%$ of the pressure rise in the air/water testing may actually be due to throat blockage. If this small effect were included in the model, the $\mathrm{K}$ versus $\alpha$ curve would shift slightly. For the RSRM propellant gas/slag system, the impact is less pronounced: a large blip $\alpha$ of 0.05 blocks $0.01 \%$ of the throat, driving only $1 / 2 \%$ of the pressure rise. This impact could be re-evaluated for tailoff events of higher $\alpha$ at lower pressure.

But the objection has been raised that the slag is not a pure liquid mass in the motor, and should be treated as a lower density grouping of gas and slag ${ }^{4}$. While this certainly seems true of the slag pool in the motor, it is an open question how that characteristic carries on when flowing through the nozzle. If the cloud is a large volume of gas with condensed particles suspended in it, then when that whole mass enters the flow, the gas from the cloud would behave just as the primary gas flow, except for composition differences. The only way to keep the slag pool gas flowing exactly with the liquid slag would be if the slag encapsulates the gas as bubbles or sponginess. This probably applies to some of the slag pool gas, but how much? Solidified slag pieces from inside full-scale motors have shown to be roughly half gas by volume, but more telling would be pieces ejected from a test motor. Even if such pieces are significantly less dense, this would represent the density at the point of solidification, at or beyond an area ratio of 4 in the nozzle exit cone. The gas in the chamber is at least 14 times denser, and the gas at the throat 8 times denser, thus occupying far less volume. Unless large bubbles of slag are found ejected from motors, this effect is not a concern. For example, a $1 / 10^{\text {th }}$ density piece would be $1 / 2$ density at the throat, driving only about $1 \%$ of the pressure rise.

\section{Further Application}

\section{A. Pressure Blip Mass Calculations}

We now turn our attention to the computation of slag mass flow rate required to produce observed pressure blips. Figure 13 shows the model's computation of pressure increase as a function of slag mass flow at typical RSRM conditions. As discussed above, the $\frac{\overline{c^{*}}}{c^{*}}$ calculation is not very sensitive to adjusting the model's parameters to match the thrust coefficient data, so the model as derived can be used where only pressure and flow rate are concerned. This is consistent with the idea that most of the shortcomings of the basic model manifest themselves aft of the throat.

Figure 14a shows the pressure blip that was

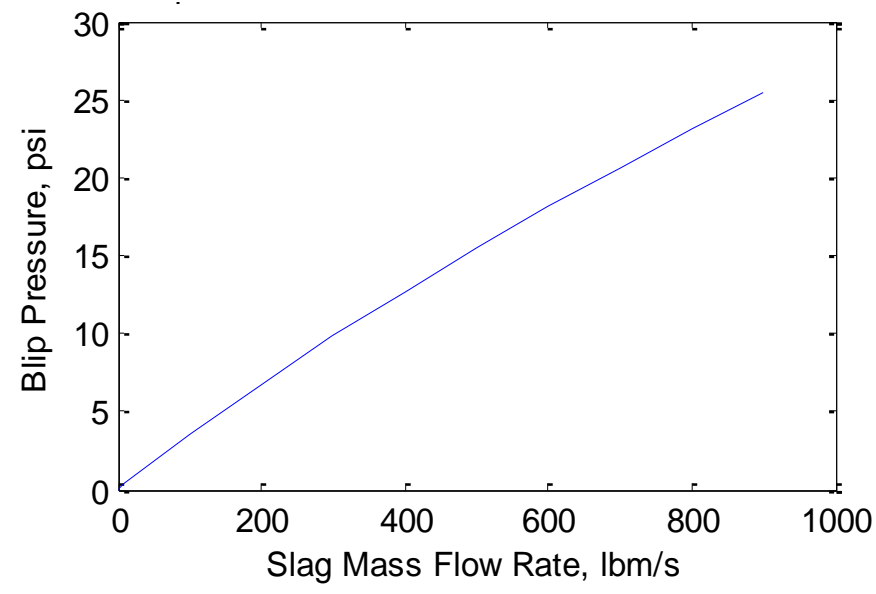

Figure 13. Approximate Slag Mass Flow Rate and Pressure Blip Magnitude for Times of Interest chiefly modeled and discussed in the blip literature. In order to calculate the slag mass flow rate causing the pressure increase, a quasi-steady assumption can be made, but the effect of the pressure rise on burn rate must be accounted for. In other words, Eq. (10) cannot be used with nominal pressure as $\mathrm{P}_{\mathrm{c} 1}$ and measured pressure as $\mathrm{P}_{\mathrm{c} 2}$, because the new condition's gas flow also deviates from the nominal gas flow. Simply enough, accounting for the burn rate merely brings into play the burn rate exponent, resulting in Eq. (18). 


$$
\frac{\overline{c^{*}}}{c^{*}}=\left(\frac{P_{c 2}}{P_{c 1}}\right)^{1-n} \frac{1}{(1+\alpha)}
$$

The condensed phase mass flow addition is buried in $c^{*}$ as $\alpha$, as shown in previous equations, and is solved for by numeric iteration.

Looking just at the steep portion of the blip, slag mass flow rate is shown corresponding to the pressure rise caused by it (Fig. 15a). Here pressure is smoothed, and the calculated total mass flowed is $243 \mathrm{lbm}$. Note the significantly different result than Whitesides's $1446 \mathrm{lbm}^{4}$.

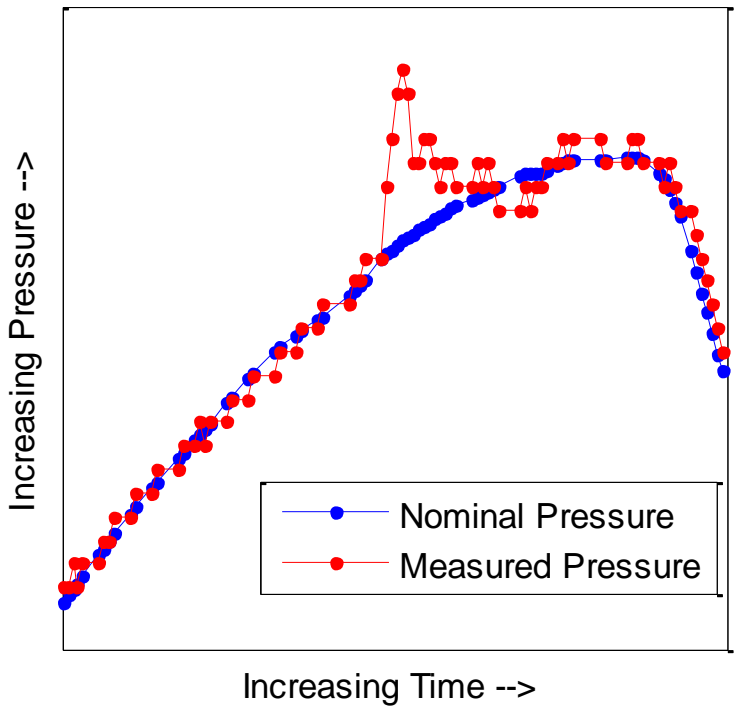

a)

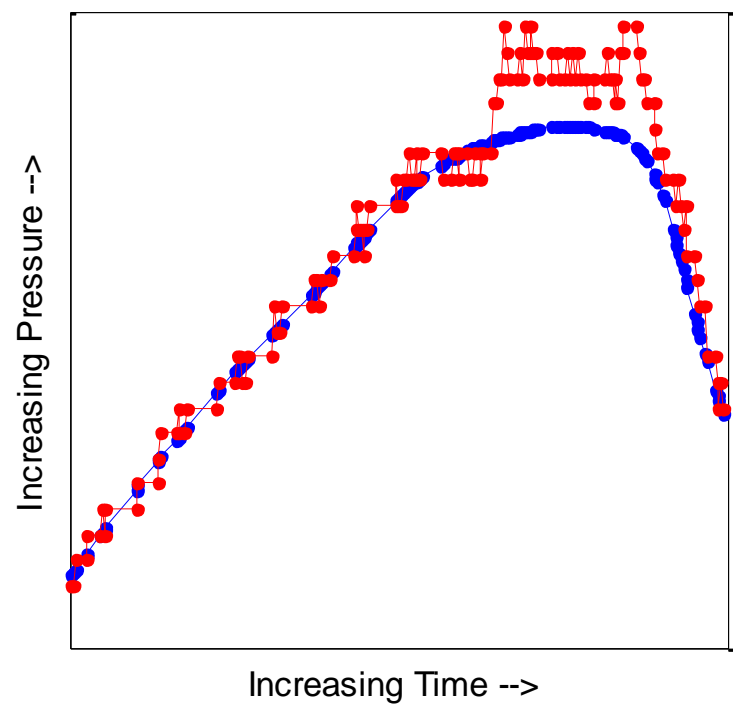

b)

Figure 14. Pressure Blip Examples: a) RSRM-29B; b) RSRM-114B
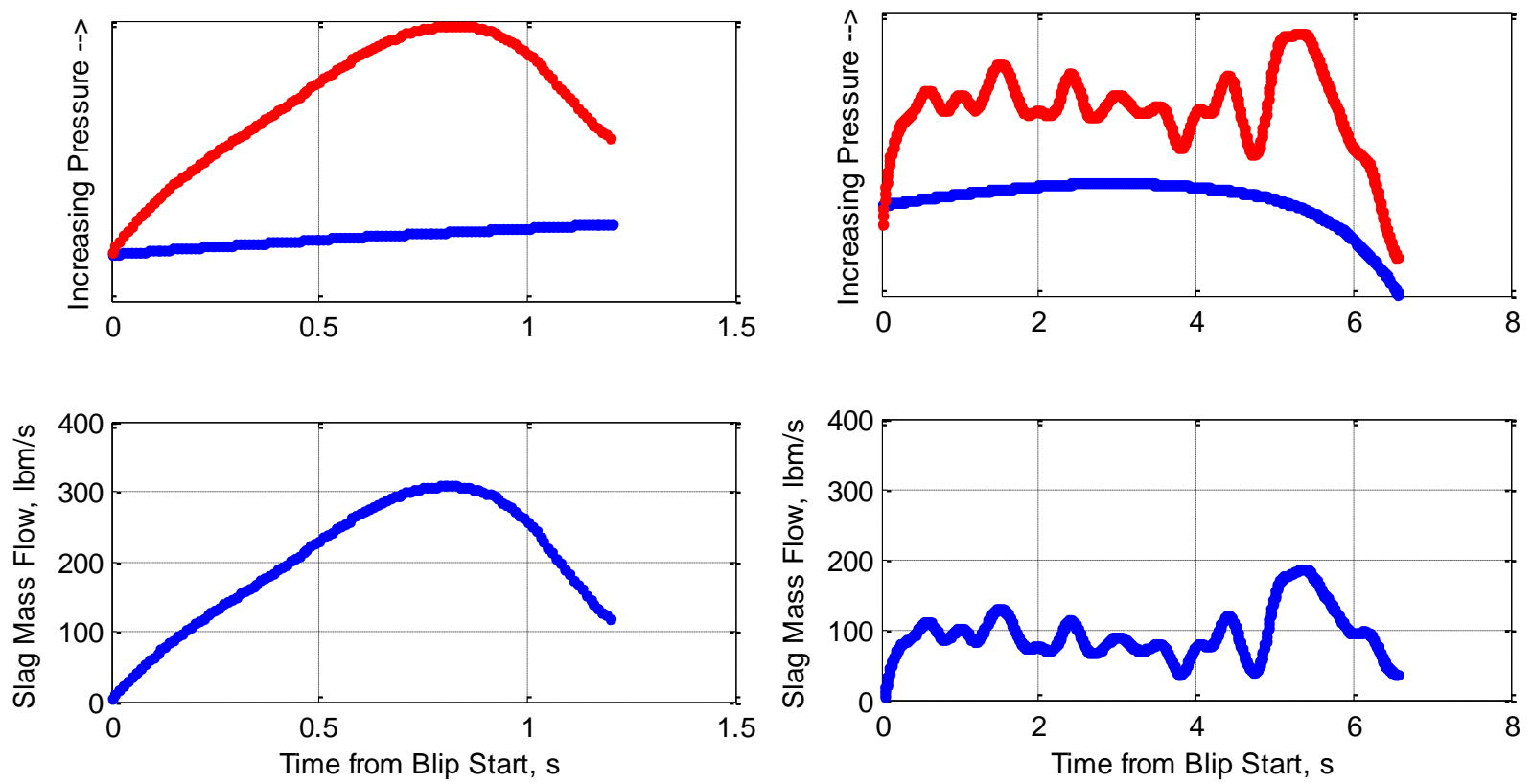

a)

b)

Figure 15. Blip Calculation Results: a) RSRM-29B; b) RSRM-114B 
As a second example, consider the last shuttle flight, motor RSRM-114B. Analysis of this differently-shaped, longer-duration event, shown in Fig. 14b and Fig. 15b, computes a total expelled slag mass of $760 \mathrm{lbm}$.

\section{B. Side Thrust}

Although this is a one-dimensional model, it can at least qualitatively describe the thrust vector effect of asymmetric slag ejection. To say that $\overline{C_{f}}>C_{f}$ is equivalent to saying that the pressure integrated through the nozzle, dotted with the axial unit vector, is greater with the condensed phase than without. This makes sense because, as has already been observed, the condensed phase slows the acceleration of the gas flow by leaching momentum from it, thus reducing the gas's expansion, which keeps its pressure from reducing as much. This matches with the depiction in Fig. 16, which agrees with Space Shuttle and Ares I-X slag disturbance and acceleration data, as demonstrated by Heim ${ }^{9}$, whose figure is copied and modified here. Due to the local increase in pressure, if slag comes out one side or zone of the nozzle, a net side force is produced in that direction. The present model can attempt to demonstrate some quantitative value here by applying the force over a nozzle sector to compute net force. An initial attempt at this does allow for the Ares I-X side force, and shows some similarity to CFD modeling for the assumption that one half of the nozzle is affected. More work would need to be done to validate if this is a useful approach for a basic side force model.

\section{Conclusion}

A one-dimensional, two-phase compressible flow model reasonably describes solid rocket motor pressure blip phenomena resulting from the ejection of material from a pool of slag during motor operation. One way to avoid knowing particle size and adding direct drag calculations is to assume similarity of the motor condition to subscale airflow testing with water injection. The condensed phase mass required to generate an observed pressure perturbation is calculated to be significantly less than previous models. This result, as well as thrust impacts, can continue to be examined based on available data from the shuttle program and other sources. If Doppler radar data of other slag expulsion events is available it would be quite helpful.

This modeling is able to shed light on motor tailoff behavior including thrust vectoring and motor pressure, and assist in more accurately predicting mass flow and mass retained versus time. These advancements in modeling will be instrumental in good flight prediction and analysis for launch vehicles using submerged nozzle rocket motors.

\section{Acknowledgments}

This work is funded under the ESTS contract with NASA Marshall Space Flight Center, Contract NNM05AB50C. The author thanks Harold Whitesides of ERC and Gene Heim and Charlie Martin of NASA for discussions, reviews, and providing data and analyses, Todd Steadman of NASA for assistance with the motor static test data, Sean Fischbach of Qualis for his review, and Jonathan Jones and Mignon Thames of NASA for their interested eye on the results.

\section{References}

${ }^{1}$ Sambamurthi, J. K., Alvarado, A., and Mathias, E. C., "Correlation of Slag Expulsion with Ballistic Anomalies in Shuttle Solid Rocket Motors," Journal of Propulsion and Power, Vol. 12, No. 4, July-August 1996, pp. 625-631.

${ }^{2}$ Hopson, C., "Space Shuttle Solid Rocket Motor Slag Expulsion Mechanisms," AIAA 95-2725, 1995.

${ }^{3}$ Martin, C., "Shuttle STS-54 Pressure Perturbation Investigation," AIAA 95-2882, 1995.

${ }^{4}$ Whitesides, R. H., Purinton, D. C., Hengel, J. E., and Skelley, S. E., "Effects of Slag Ejection on Solid Rocket Motor Performance," AIAA 95-2724, 1995. 
${ }^{5}$ Dotson, K. W., Murdock, J. W., and Kamimoto, D. K., "Launch Vehicle Dynamic and Control Effects from Solid Rocket Motor Slag Ejection," Journal of Propulsion and Power, Vol. 15, No. 3, May-June 1999, pp. 468-475.

${ }^{6}$ Kliegel, J. R., One Dimensional Flow of a Gas Particle System, Space Technology Laboratories, Inc., TR-59-0000-00746, Los Angeles, 8 July 1959.

${ }^{7}$ Dunn, S. S., Coats, D. E., and French, J. C., SPP'04 Standard Stability Prediction Method for Solid Rocket Motors Axial Mode Computer Program User's Manual, Software \& Engineering Associates, Inc., Carson City, 2005, pg. 2-2.

${ }^{8}$ Schetz, J. A., Boundary Layer Analysis Revised, AIAA Education Series, AIAA, Reston, VA, 2010, pp. 35-37.

${ }^{9}$ Heim, E., "Ares I-X Flight Dynamics During Solid Rocket Motor Thrust Tail-Off," $58^{\text {th }}$ JANNAF Propulsion Meeting Papers on Disc [CD-ROM], CPIAC, Greenbelt, MD, April 2011. 